\title{
BRAHMA $^{+}$: A Framework for Resource Scaling of Streaming and ASAP Time-Varying Workflows
}

\author{
Ankita Atrey ${ }^{(\mathbb{1}}$, Gregory Van Seghbroeck, Bruno Volckaert, and Filip De Turck ${ }^{(\mathbb{1}}$
}

\begin{abstract}
Automatic scaling of complex software-as-a-service 2 application workflows is one of the most important problems con3 cerning resource management in clouds. In this paper, we study 4 the automatic workflow resource scaling problem for streaming 5 and ASAP workflows, and its time-varying variant where the 6 workflow resource requirements change over time. Service com7 ponents of streaming workflows execute concurrently while those 8 of ASAP workflows execute sequentially. We propose an intelli9 gent framework, BRAHMA $^{+}$, which possesses the capability to learn the workflow behavior and construct a knowledge base that 11 serves as its decision making engine. The proposed resource pro12 visioning algorithms leverage this learned information curated in 13 the knowledge base to perform informed and intelligent scaling 14 decisions. Additionally, BRAHMA ${ }^{+}$employs the use of online15 learning strategies to keep the knowledge base up-to-date, thereby 16 accommodating the changes in the workflow resource require17 ments over time. We evaluate the proposed algorithms using ${ }_{18}$ CloudSim simulations. Results on streaming and ASAP work19 flows, with both static and time-varying resource requirements 20 show that the proposed algorithms are effective and produce good 21 cost-quality trade-offs. The proactive and hybrid algorithms meet 22 the service level agreements and restrict deadline violations to a 23 small fraction (3\%-5\% in the considered scenarios), while only 24 suffering a marginal increase in average cost per component 25 compared to the described baseline algorithms.
\end{abstract}

Index Terms-Cloud resource provisioning, workflows, 27 cloud scalability, adaptive clustering, knowledge base, 28 deadline-constraints, SLA, cloud simulation.

29

30

31 32 Cloud users enjoy flexible and cost-effective usage of variзз ous cloud services, however, providing quality of service 34 meeting SLAs, scalability, and deadline constraints - while 35 maintaining cost-effectiveness with highly dynamic resource 36 requirements exhibited by end-user requests, is the paramount 37 concern of various service providers.

38 Having said that, resource management continues to be one 39 of the most fundamental and important areas of research in 40 the field of cloud, distributed, and grid computing. While

Manuscript received June 26, 2017; revised December 23, 2017 and April 9, 2018; accepted April 15, 2018. This research is partly funded by the IWT SBO DeCoMAdS project. The associate editor coordinating the review of this paper and approving it for publication was L. Y. Chen. (Corresponding author: Ankita Atrey).

The authors are with the Internet Technology and Data Science Laboratory, Ghent University-imec, 9052 Gent, Belgium (e-mail: ankita.atrey@ugent.be gregory.vanseghbroeck@ugent.be; bruno.volckaert@ugent.be; filip.deturck@ ugent.be).

Digital Object Identifier 10.1109/TNSM.2018.2830311

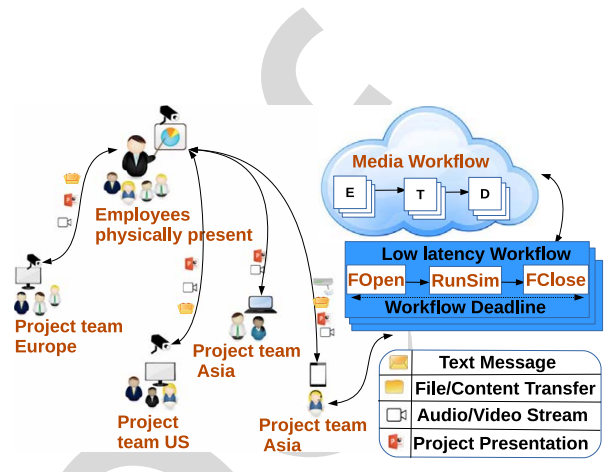

Fig. 1. Use case: An online collaborative meeting room service.

there exists a plethora of research in devising industry ${ }_{41}$ scale resource management systems especially by Internet 42 giants like Google [42], Facebook [5], Microsoft [10], [21], Alibaba [48] etc., the focus of these systems have been on execution environments like grids and clusters, and such efforts have been relatively scarce for SaaS application workflows in cloud-based systems [22]. In this article, we propose a unified framework, BRAHMA ${ }^{+}$, for resource scaling in clouds.

\section{A. Use Case: Online Collaborative Meeting}

The use case under investigation (Fig. 1) is an elastic, 50 multi-tenant online meeting room offering an interactive col- 51 laboration service. This use case is inspired by the EMD 52 project [4], which investigates scalable $\mathrm{A} / \mathrm{V}$ collaboration applications (streams) and deadline-critical jobs (such as decision support, data analysis, etc.) triggered by the end-user ${ }_{55}$ during these collaborations. The project leader is presenting 56 an interactive media (A/V streaming) session, where some ${ }_{57}$ colleagues are present in the meeting room, while others are 58 connected remotely. Every stream consists of an encoder, a 59 transcoder and a decoder, all of which have different SLA 60 requirements in an attempt to provide a flawless service (no ${ }_{61}$ $\mathrm{A} / \mathrm{V}$ interruptions, stuttering, etc.). The A/V stream encapsu- 62 lates a streaming workflow (Definition 4), which is similar 63 to the long-running services presented in Fig. 2 that should 64 not experience any downtime. Each attendee can addition- 65 ally trigger low-latency/deadline-critical workflows during the 66 meeting to, e.g., run analytical simulations (file-open $\rightarrow$ run- ${ }_{67}$ simulation $\rightarrow$ file-close workflow in Fig. 1) and show the results to the meeting audience or render high quality graphics. We use the term ASAP workflows (Definition 7) to denote these low latency jobs. While streaming workflows usually constitute one or more service components executing concurrently, 72 the components of ASAP workflows are executed sequentially, 73 


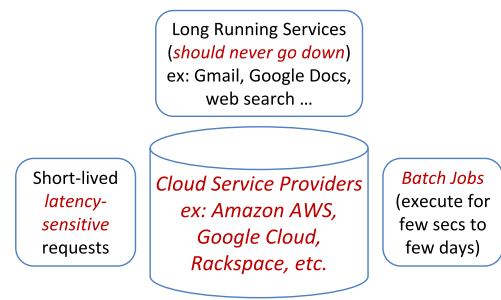

Fig. 2. Types of jobs in cluster/grid/cloud computing environments [42].

74 thereby exhibiting runtime characteristics which differ from 75 that of the former. Users can join or leave these online 76 meetings at any point in time, leading to potentially large fluc77 tuations in terms of number of tenants using the system, and 78 each user can trigger multiple ASAP workflows during the 79 session.

80 In the context of the use-case discussed above, meeting the 81 strict deadline constraints of ASAP workflows and SLAs of 82 streaming workflows (for A/V quality), while keeping cloud 83 resource cost as low as possible, is the focus of this work. This 84 scenario presents a plethora of challenges stated as follows:

85 (1) Scaling the resulting application up or down in order 86 to keep the SLAs, no longer becomes an issue of scaling 87 resources for a single service, but instead results in a com88 plex problem of scaling all individual service endpoints in the 89 workflow, depending on their runtime monitored behavior [9]. 90 (2) As described above, heterogeneity of application work91 flows (Fig. 2) leads to a host of characteristics: ranging from 92 execution flows being either sequential or concurrent [8] to the 93 nature of the deadlines associated with the workflows being 94 either strict or fuzzy. (3) The resource requirements of the jobs 95 submitted to a cloud environment are usually not static. Rather, 96 in real-world cloud environments the type of workflows sub97 mitted by users and their resource requirements change over 98 time [31], [47]. For instance, given the meeting room use-case, 99 a user might trigger a high-quality graphic render as an ASAP 100 workflow during a weekly-update meeting, while during a 101 technical deep-dive session, she might trigger an analytical 102 simulation. Thus, the resource management algorithms should 103 be adaptive thereby enabling effective resource provisioning 104 for such time-varying workflows.

105 To effectively address the challenges besetting the resource 106 management problem in real-world scenarios for clouds, there 107 is a need for a framework, tailored to serving cloud service 108 workflow requests, that (1) possesses the capability to han109 dle different types of workflows, (2) intelligently performs 110 resource provisioning tasks under specified deadlines or SLAs, 111 and (3) possesses algorithms that adapt to the temporally 112 changing resource requirements posed by these workflows.

${ }_{113}$ This article presents a framework, $B R A H M A^{+}$, which 114 incorporates the use of mathematical models (classifica115 tion and clustering) to learn the workflow behavior and 116 curates a knowledge base $(K B)$ that aids in taking informed 117 future resource provisioning decisions. These models anal118 yse the resource request patterns of workflows to predict 119 whether a new workflow will meet its deadline or not, 120 and clusters the workflows into groups possessing similar 121 resource requirements. Moreover, for time-varying workflows 122 we design and implement online versions of the proposed learning algorithms, where the learned models are updated 123 with temporally changing data. To further enhance the effi- 124 cacy and efficiency of the time-aware learning process, we 125 use a sliding window, which controls the amount of historical 126 data to be used for learning at any given time instant, thereby 127 improving both the quality (helps ignoring irrelevant historical 128 data) and efficiency (learning from a relevant fraction of the 129 complete data).

In sum, in this article we address the automatic workflow 131 resource scaling problem (Section III) under the combined 132 presence of streaming and ASAP workflows, called AWS-SA, 133 and its time-varying variant, called $A W S$-t $S A$ where the work- 134 flow resource requirements change over time. 135

Key contributions are as follows: 136

- A novel framework, BRAHMA+ (Section IV), which 137 learns workflow behaviour over time and stores this infor- 138 mation in a KB. BRAHMA ${ }^{+}$possesses the capability to ${ }_{139}$ predict workflow deadlines and cluster these workflows 140 into semantically meaningful groups. Additionally, the ${ }_{141}$ online learning algorithms of $\mathrm{BRAHMA}^{+}$are capable 142 of adapting to the changes in resource request patterns 143 exhibited by time-varying workflows. 144

- Resource provisioning algorithms (Section V) that lever- 145 age BRAHMA $^{+}$to maintain SLAs and deadlines for 146 streaming and ASAP workflows respectively, as well as 147 their time-varying variant, while keeping the cost in line. 148

- Empirical analysis (Section VII) portraying the effective- 149 ness of the proposed algorithms. Our algorithms keep 150 the SLAs and restrict deadline violations to 3-5\%, while 151 only suffering a marginal increase in the average resource 152 utilization cost of $5-8 \%$ over the baselines.

\section{RELATED WORK}

154

Resource management and scheduling [22] is a fundamental 155 and one of the most extensively studied problems in the field of 156 cloud computing. Here, we provide an overview of the existing 157 works that overlap with the work presented in this article. 158

Workflows provide a natural and attractive choice for repre- 159 senting a host of SaaS applications, thus, automatic workflow 160 resource scaling and scheduling [13] with focus on main- 161 taining quality of service parameters, like SLAs [29] and 162 deadline-constraints [6], [38], has been a hot topic of research 163 in the broad area of cloud resource management. The readers 164 are referred to [43] for a detailed and recent survey. $\quad 165$

SLA-aware resource provisioning: focusses on strategies for 166 resource scaling to keep the SLAs in line while minimiz- 167 ing cost. Wu et al. [44], [45], presented SLA-aware resource 168 provisioning algorithms for SaaS providers. The authors pro- 169 pose maximum and minimum available space based resource 170 reservation and request rescheduling strategies, while using 171 customer profiles to handle dynamic and changing customer 172 requests. Later, the authors developed a method for admis- 173 sion control of user requests [46], thus facilitating prevention 174 of additional user requests that would lead to SLA violations 175 from being accepted.

176

Serrano et al. presented a new model: SLA aware service 177 (SLAaaS) [37], proposed a language for describing SLAs and 178 an approach using control-theory for keeping the SLA of cloud 179 applications. Focussing on application workflows, Atrey et al. 180 
181 proposed a pro-active algorithm [9] that uses a monitoring 182 mechanism to track the run time behavior of each work183 flow component and horizontally scales resources accordingly, 184 thereby avoiding SLA violations. Singh et al. [39] studied the 185 effect of various QoS parameters on the rate of SLA violations, 186 and proposed an autonomic pipeline along with a knowledge 187 store to devise effective resource provisioning strategies.

188 In addition to research on devising strategies for SLA-aware 189 resource provisioning, a few studies have performed bench190 marking and validation [7], [17] of various SLA-aware models 191 and resource provisioning algorithms.

192 Deadline-aware resource provisioning: focusses on devising 193 strategies to minimize the deadline violations of jobs submit194 ted to clouds. Genez et al. [16] present an Integer Linear 195 Programming (ILP) based algorithm for scheduling SaaS 196 workflows in IaaS clouds, which finds the mapping between 197 workflow tasks and VMs provided by the IaaS providers to 198 minimize the overall cost and achieve deadline constraints.

199 Poola et al. present robust and fault-tolerant resource 200 scheduling algorithms with three multi-objective 201 resource allocation policies in [31]. Moving ahead, 202 Rodriguez and Buyya [34] applied a genetic algorithm 203 (Particle Swarm Optimization) in order to obtain an opti204 mized solution in terms of cost, deadline and elasticity, 205 highlighting resource provisioning techniques for scientific 206 workflows on IaaS. Luo et al. propose a resource provisioning 207 algorithm [26] for hybrid settings comprising both grids and 208 clouds. The idea is to estimate the probability of deadline 209 violation of a sub-task in a workflow, and then later redirect 210 certain sub-tasks from grids to intelligently selected virtual 211 resources on clouds, in order to achieve strict workflow 212 deadline-constraints. Recently, Atrey et al. [8] presented a 213 framework called BRAHMA (which has been significantly 214 extended to $\mathrm{BRAHMA}^{+}$in this article) that used workflow 215 clustering and a curated $\mathrm{KB}$ to perform resource provisioning 216 for workflows with strict deadline-constraints.

217 Resource provisioning using machine learning: is a rela218 tively recent paradigm in clouds, where researchers have incor219 porated the use of various classification and clustering methods 220 for learning and characterizing workflow behaviour [23], [25]. 221 Mon et al. [28] proposed workflow clustering based on 222 task dependencies with an aim to minimize the data trans223 fer overhead of data-intensive scientific workflows. Moving 224 ahead, Peng et al. [30] presented a machine learning frame225 work that used a radial basis function based neural net226 work to estimate application resource requirements, and a $227 \mathrm{k}$-means based genetic clustering algorithm for performing 228 multi-objective optimization to solve the resource provision229 ing problem. Atrey et al. [8] proposed a machine learning 230 framework that curates all the learned information in a $\mathrm{KB}$. ${ }_{231}$ Very recently, Li et al. [24] present a resource scheduling 232 algorithm that uses fuzzy clustering methods to identify dif233 ferent resource clusters thereby simplifying their allocation 234 to jobs.

235 Resource provisioning for dynamic workflows: addresses 236 workflows with dynamically changing resource requirements. 237 To the best of our knowledge, research in this context has 238 been scarce. Zhang et al. [47] presented ROSA, an online 239 randomized algorithm that stacks the execution of multiple
TABLE I

SUMMARY OF NOTATIONS USED

\begin{tabular}{|c|c|}
\hline Item & Definition \\
\hline $\mathcal{V}$ & The pool of VMs; $\forall i, V_{i} \in \mathcal{V}$. \\
\hline $\mathcal{W}_{s}$ & Set of streaming workflow requests. \\
\hline $\mathcal{W}_{a}$ & Set of ASAP workflow requests. \\
\hline $\mathcal{W}$ & Set of workflow requests; $\forall j, W_{j} \in \mathcal{W}=\mathcal{W}_{s} \cup \mathcal{W}_{a}$ \\
\hline $\mathcal{D}_{s}(t)$ & Time varying distribution for streaming worfklow requests. \\
\hline $\mathcal{D}_{a}(t)$ & Time varying distribution for ASAP worfklow requests. \\
\hline$C_{k j}$ & A service component for the workflow $W_{j} ; \forall k, C_{k j} \in W_{j}$ \\
\hline$M I_{C_{k j}}$ & The number of instructions (in MI) required to execute $C_{k j}$. \\
\hline$D C_{W_{j}}$ & The deadline constraint of $W_{j} \in \mathcal{W}_{a}$ \\
\hline$S L A_{\text {status }}^{C_{k j}}$ & The status (binary) of the SLA of $C_{k j}$, i.e. met or violated. \\
\hline DEADLINE $E_{\text {status }}^{W_{j}}$ & The status (binary) of the Deadline of $W_{j}$, i.e. met or violated. \\
\hline$\eta$ & Fraction of ASAP workflows with violated deadlines. \\
\hline$M I P S_{i}$ & The processing power in MIPS of VM $V_{i}$ \\
\hline$C C$ & Set of identified clusters and their cluster centers, $\rho=|C C|$. \\
\hline$C M$ & Classification model. \\
\hline $\mathcal{N}_{\text {running }}^{i}$ & Number of components currently running on VM $V_{i}$. \\
\hline $\mathcal{N}_{\max }^{i}$ & Maximum number of components allowed on VM $V_{i}$ \\
\hline$\tau$ & $\begin{array}{l}\text { Parameter controlling how quickly the Proactive algorithm } \\
\text { intervenes in terms of scaling resources up or down. }\end{array}$ \\
\hline$s w\left[s w_{s t a r t}, s w_{\text {end }}\right]$ & $\begin{array}{l}\text { The sliding window } s w \text { with window size }=\left|s w_{\text {end }}-s w_{\text {start }}\right| \text {. } \\
\text { The rate of information decay over time. }\end{array}$ \\
\hline$T_{\text {reserve }}^{V_{i}}$ & Time required for reservation of a new VM $V_{i}$ \\
\hline$T_{\text {migrate }}$ & Time required for migrating a service component to $V_{i}$. \\
\hline $\begin{array}{l}P_{\text {reserve }}^{V_{i}} \\
P_{\text {migrate }}\end{array}$ & $\begin{array}{l}\text { Penalty incurred due to reservation of a new VM } V_{i} \text {. } \\
\text { Penalty incurred owing to migrating components to } V_{i} \text {. }\end{array}$ \\
\hline
\end{tabular}

jobs submitted to the cloud environment, thereby achieving 240 spatial multiplexing. This helps minimize cost with the capa- 241 bility to leverage volume discounts offered by cloud service 242 providers, while also keeping the job-level constraints in 243 line. Poola et al. [32] presented an adaptive resource pro- 244 visioning algorithm that is capable of incorporating the use 245 of both spot and on-demand instances, thereby minimizing 246 the total cost: as it leverages the price benefit from spot 247 instances, and ensuring fault-tolerance by meeting workflow 248 level deadlines using on-demand instances whenever neces- 249 sary. Recently, adaptive resource scheduling [14] has also 250 been studied in the context of software defined networks [41]. 251 Rodriguez and Buyya [35] proposed a container-based algo- 252 rithm that can adapt to changes in the workload, while 253 mitigating inefficiencies in resource utilization and meeting 254 workflow level deadlines.

Despite wide-spread research in the broad area of workflow 256 resource scheduling [43], to the best of our knowledge, none 257 of the existing state-of-the-art methods are capable of solving 258 this problem in a holistic manner. Specifically, the existing 259 works have focused on the two problems, i.e., scaling stream- 260 ing and deadline-critical workflows independently, however, 261 a unified and generic framework possessing capabilities of 262 collectively scaling both types of workflows is non-existent. 263 Additionally, research on devising algorithms that adapt to 264 temporally changing resource requirements of workflows has 265 been scarce. To this end, this article presents an enabling, uni- 266 fied, and adaptive framework, BRAHMA ${ }^{+}$, with algorithms 267 for provisioning cloud resources to streaming (SLA-aware) 268 and ASAP workflows (with strict deadline-constraints), whose 269 resource requirements change over time.

\section{PRoblem Statement}

271

This section provides a concise model of streaming and 272 ASAP workflows, with an introduction of their basic con- 273 cepts followed by a formal description of the AWS-SA problem, 274 
275 and its variant $A W S$ - $t S A$, focussing on time-varying workflows. 276 Table I summarizes the notations used in the rest of the article. 277 Definition 1 (Resource): A resource corresponds to a pro278 cessing unit with specifications defined in terms of processing 279 power (in MIPS), memory (in GB), storage (in GB), and 280 network bandwidth (in Mbps).

281 Cloud computing environments offer virtual resources in the 282 form of virtual machines (VMs), containers etc. In this study, 283 we consider a pool of resources called a VM pool $\forall i, V_{i} \in \mathcal{V}$. 284 Since cloud-based applications are usually built as work285 flows integrating multiple existing services (albeit with custom 286 glue code tying all of them together), an application workflow 287 is defined as follows:

Definition 2 (Application Workflow): An application work289 flow $W_{j}(C, E)$ is defined as a directed acyclic graph (DAG) 290 comprising a set of service components $C_{j}$ and a set of 291 edges $E_{j}$. Each component $\forall k, C_{k j} \in C_{j}$ represents an atomic 292 task in the application workflow $W_{j}$, while each directed edge 29з $\forall k, l, e=\left(C_{k j}, C_{l j}\right) \in E_{j}$ defines the dependency of the ${ }_{294}$ component $C_{l j}$ on component $C_{k j}$.

295 Definition 3 (Workflow Resource Requirements): The 296 resources required by a component $C_{k j}$ of a workflow $W_{j}$ 297 are defined in terms of number of instructions to be executed 298 (measured in millions of instructions $\left(M I_{C_{k j}}\right)$ ), the memory 299 and storage space required $\left(\left(\mathrm{Mem}_{C_{k j}}\right)\right.$ in $\left.\mathrm{GB}\right)$, and the 300 number of bits to be transferred over the network $\left(\left(B W_{C_{k j}}\right)\right.$ 301 measured in millions of bits (Mb)).

Application workflows can possess a wide-variety of char303 acteristics in terms of execution flow, resource requirements 304 etc. Based on these characteristics we next define the two types 305 of workflows considered in this article.

306 Definition 4 (Streaming Workflow): A streaming workflow ${ }_{307} W_{j} \in \mathcal{W}_{s}$ is an application workflow where service compo308 nents $C_{l j} \in C_{j}$ continuously receive streaming data from other 309 components $C_{k j} \in C_{j}$ via directed edges $e=\left(C_{k j}, C_{l j}\right) \in$ ${ }_{310} E_{j}$, while they themselves stream their output data to other 311 workflow components following their execution.

312 For instance, if the workflow in Fig. 3 is a streaming work313 flow, $C_{21}$ would continuously stream output data to $C_{31}$ and ${ }_{314} C_{41}$, who in turn would process that input data and stream it 315 to $C_{51}$. All service components are hence processing informa316 tion in parallel. Each streaming workflow service component 317 possesses a separate SLA, which defines its minimal resource 318 requirements (in terms of processing power, memory, storage 319 etc.) to ensure proper working of the workflow according to its 320 specifications. Using this component-level minimal resource 321 requirement and the resource specification (processing power, 322 memory, storage etc.) of the assigned VM $V_{i} \in \mathcal{V}$, we define ${ }_{323}$ the maximum number of components $\mathcal{N}_{\max }^{i}$ that can simulta${ }_{324}$ neously run on $\mathcal{V}_{i}$ while ensuring SLAs are met. For example, 325 given a VM with processing power as 1500 MIPS and the 326 minimal resource requirement per component to be $50 \mathrm{MI}$, 327 the maximum number of components that can be scheduled 328 on this VM is 30 . Note that for simplicity each VM is assigned 329 to components of one specific type, i.e., those possessing the ${ }_{330}$ same minimal resource requirement. Using $\mathcal{N}_{\max }^{i}$ we further 331 define the SLA status of a component and the average SLA 332 violation duration.

${ }_{333}$ Definition 5 (SLA Status): The SLA status for each service ${ }_{334}$ component $C_{k j}$ of a workflow $W_{j}$ running on a $\mathrm{VM} V_{i}$ is

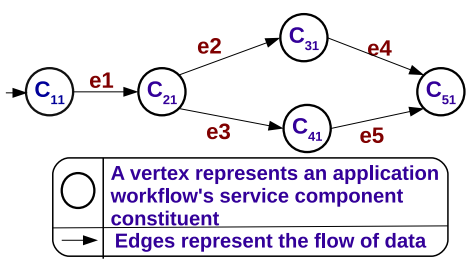

Fig. 3. An application workflow $W_{1}$ composed of multiple service components and inter-component data flows.

defined as a binary variable which assumes the value of false 335 if the SLAs are violated, or true otherwise. Mathematically, ${ }_{336}$

$$
S L A_{\text {status }}^{C_{k j}}= \begin{cases}\text { false, } & \text { if } \mathcal{N}_{\text {running }}^{i}>\mathcal{N}_{\text {max }}^{i} \\ \text { true, } & \text { otherwise }\end{cases}
$$

where, $\mathcal{N}_{\text {running }}^{i}$ denotes the number of components currently $3 з 8$ running on the VM $V_{i}$.

339

Definition 6 (Average SLA Violation Duration): The SLA 340 violation duration of a service component $C_{k j}$ is defined as 341 the amount of time for which its $S L A_{\text {status }}^{C_{k j}}$ is violated over 342 its runtime duration. Thus, for a simulation with $w$ workflow 343 requests $W_{j} \in \mathcal{W} \mid 1 \leq j \leq w, c_{j}$ service components 344 $C_{k j} \in W_{j} \mid 1 \leq k \leq c_{j}$, and $T_{\text {slaviolate }}^{C_{k j}}$ being the duration 345 for which the SLAs remain violated for a component $C_{k j}$, we 346 mathematically state the average SLA violation duration as: 347

$$
\frac{1}{w}\left(\sum_{j=1}^{w}\left(\frac{1}{c_{j}} \sum_{k=1}^{c_{j}}\left(T_{\text {slaviolate }}^{C_{k j}}\right)\right)\right) .
$$

Definition 7 (ASAP Workflow): An ASAP workflow 349 $W_{j} \in \mathcal{W}_{a}$ is an application workflow where the execu- 350 tion flow between service components is sequential. More 351 specifically, the execution control moves from one component 352 $C_{k j} \in C_{j}$ to the subsequent workflow component(s) $C_{l j} \in C_{j}$, 353 once the former finishes processing thereby passing its full 354 output to the latter.

Again as an example, if the workflow in Fig. 3 would 356 be sequential, $C_{21}$ would, once it has finished processing, 357 send all its output data in parallel along the edges $e 2$ and 358 $e 3$ to $C_{31}$ and $C_{41}$ respectively. At that point in time, $C_{31} 359$ and $C_{41}$ start processing. Additionally, each ASAP work- 360 flow possesses a deadline-constraint $\left(D C_{\mathcal{W}_{j}}\right)$ which is used 361 to identify a VM $\mathcal{V}_{i}$ that possesses the desired resources 362 to ensure proper working of the workflow according to its 363 specifications.

Definition 8 (Deadline Status): The deadline status of a 365 workflow $W_{j}$, running on a VM $V_{i}$, is defined as a binary 366 variable which assumes the value of false if its deadline- 367 constraint $D C_{\mathcal{W}_{j}}$ is not met, and true otherwise. The fraction 368 of the workflows whose deadlines are violated is denoted by 369 $\eta$. Mathematically,

370

$$
\eta=\frac{1}{w}\left(\sum_{j=1}^{w} I\right)
$$

where $I$ is $W_{j}$ the indicator function: $I=1$ if 372 DEADLINE ${ }_{\text {status }}^{W_{j}}=$ false; and 0 otherwise.

Definition 9 (VM Cost): VM cost is defined as the sum of 374 all costs related to resource usage when running streaming 375 
376 and ASAP workflow service components. Thus, for a sim377 ulation with $w$ workflow requests, each one with $c_{j}$ service 378 components, and $M_{k j}, S_{k j}, C P U_{k j}$, representing, memory, 379 storage and CPU costs respectively for a component $C_{k j}$, 380 we mathematically define VM cost and average VM cost as 381 follows:

383

$$
\begin{aligned}
& \sum_{j=1}^{w}\left(\sum_{k=1}^{c_{j}}\left(M_{k j}+S_{k j}+C P U_{k j}\right)\right) \\
& \frac{1}{w}\left(\sum_{j=1}^{w}\left(\sum_{k=1}^{c_{j}}\left(M_{k j}+S_{k j}+C P U_{k j}\right)\right)\right) .
\end{aligned}
$$

384 Definition 10 (Penalty): The Penalty is the cost spent on 385 components, while waiting for (1) a new VM reservation $386 P_{\text {reserve }}$ and (2) migration of components from one VM to 387 another $P_{\text {migrate }}$. We mathematically state the Penalty and 388 the average Penalty as follows:

389

$$
\begin{aligned}
& \sum_{j=1}^{w}\left(\sum_{k=1}^{c_{j}}\left(P_{\text {reserve } k j_{j}}+P_{\text {migrate }_{k j}}\right)\right) \\
& \frac{1}{w}\left(\sum_{j=1}^{w}\left(\frac{1}{c_{j}} \sum_{k=1}^{c_{j}}\left(P_{\text {reserve }_{k j}}+P_{\text {migrate }_{k j}}\right)\right)\right)
\end{aligned}
$$

391 The technical problem studied in this work is inspired by 392 the use case of online collaborative A/V meetings where both 393 streaming and ASAP workflows co-exist. Note that tenant 394 requests for streaming and ASAP workflows follow time${ }_{395}$ varying distributions $\mathcal{D}_{s}(t)$ and $\mathcal{D}_{a}(t)$ respectively. While 396 streaming workflows do not benefit from assigning more 397 resources to them than required, as one cannot 'speed up' 398 an online collaborative meeting, ASAP workflows definitely 399 benefit from finishing early (meeting their deadlines), when 400 allocated to more powerful resources. Owing to this significant 401 difference in characteristics, scaling the service end-points of 402 applications where streaming and ASAP workflows co-exist is 403 a challenging problem. We name this problem $A W S-S A$, and 404 formally define it as:

405 Problem 1 (AWS-SA): Given a VM pool $\mathcal{V}$, a set of work406 flow requests $(\mathcal{W})$ consisting of a combination of streaming ${ }_{407}\left(\mathcal{W}_{S}\right)$ and $\operatorname{ASAP}\left(\mathcal{W}_{a}\right)$ workflow requests, following time 408 varying distributions $\mathcal{D}_{s}(t)$ and $\mathcal{D}_{a}(t)$ respectively, the maxi409 mum number of allowed requests $\left(\mathcal{N}_{\max }^{i}\right)$ and the processing 410 power $\left(M I P S_{i}\right)$ for each VM $\left(\forall V_{i} \in \mathcal{V}\right)$, perform automatic ${ }_{411}$ resource provisioning to keep the SLAs $\left(S L A_{\text {status }}^{C_{k j}}=\right.$ true $)$ 412 and the deadline-constraints $\left(D E A D L I N E_{\text {status }}^{W_{j}}=\right.$ true $)$ for 413 all the workflow components $C_{k j} \mid \forall k, C_{k j} \in W_{j}, \forall j, W_{j} \in$ ${ }_{414} \mathcal{W}$, while simultaneously minimizing the vm cost and penalty.

415 In addition to the number of streaming and ASAP workflows ${ }_{416}$ changing over time (denoted by $\mathcal{D}_{s}(t)$ and $\mathcal{D}_{a}(t)$ ), the actual 417 resource requirements of workflows change as well. To this 418 end, we address the temporal variant of the AWS-SA problem 419 called the $A W S$ - $t S A$ problem.

420 Problem 2 (AWS- $t S A)$ : The AWS-SA problem where the 421 resource requirements $\left(M I_{C_{k j}}, M e m_{C_{k j}}, B W_{C_{k j}}\right)$ of workflow 422 components, $C_{k j} \mid \forall k, C_{k j} \in W_{j}, \forall j, W_{j} \in \mathcal{W}$, change over 423 time.
IV. BRAHMA ${ }^{+}$FRAMEWORK

424

In this section, we present the BRAHMA $^{+}$framework 425 and provide a description of its core components. In this 426 article, BRAHMA [8] has been significantly extended using 427 online learning strategies as $\mathrm{BRAHMA}^{+}$to learn workflow ${ }_{428}$ request behavior in an online manner, i.e., without the need for 429 training data to bootstrap the learning process. This enables 430 $\mathrm{BRAHMA}^{+}$to handle workflows whose resource require- 431 ments change over time. We also provide insights about the 432 way in which BRAHMA $^{+}$facilitates development of effec- 433 tive resource provisioning strategies. The building blocks of 434 the $\mathrm{BRAHMA}^{+}$framework are detailed next. 435

- Classification (Build Classifier): analyses the resource 436 requirements and request patterns exhibited by ASAP ${ }_{437}$ workflows, and learns a decision boundary, using histori- 438 cal resource requirement data of workflows submitted to 439 a cloud environment, capable of predicting whether the 440 deadline of a previously unseen workflow would be met ${ }_{441}$ or violated. The main benefit that the classifier module 442 provides is the ability to predict the DEADLINE $E_{\text {status }} 443$ of (previously unseen) incoming ASAP workflows, facil- 444 itating more informed resource provisioning decisions. $\quad 445$

- Clustering (Identify Clusters): allows for fine-grained 446 analysis of the behaviour exhibited by ASAP work- 447 flows. Here, the resource request patterns are clus- 448 tered, thereby creating semantically meaningful groups 449 of ASAP workflows, with each group possessing sim- 450 ilar resource requirements. The advantage of clustering 451 is that once these clusters are identified, it is easier to 452 devise customized and informed resource provisioning 453 strategies pertaining to each cluster. Moving ahead, any 454 previously unseen ASAP workflow request can then be 455 assigned to its most similar group, and hence utilize the 456 already devised resource provisioning strategy for that 457 group.

- Online Clustering: extends the clustering module by mak- 459 ing it flexible and adaptive to effectively accommodate 460 changes in data distributions originating from time- 461 varying workflows. More specifically, since the resource 462 requirements of ASAP workflows may change over time, 463 the identified clusters have to change as well, as the clus- 464 ters generated from older data will have been invalidated. 465 Unlike conventional methods, where clustering is per- 466 formed as a single-shot process comprising two steps: (1) 467 cluster identification, and (2) cluster assignment; online 468 clustering methods continuously learn from the data, i.e., 469 the identified clusters are refined as and when newer 470 data-points are ingested by the system. Moreover, to 471 ensure consistency the identified clusters are updated 472 regularly in the knowledge base.

473

- Knowledge Base (KB): is one of the most important com- 474 ponents of the $\mathrm{BRAHMA}^{+}$framework as it curates all 475 the information learned from the classifier and the cluster- 476 ing modules. More specifically, the KB stores an updated 477 copy of the classifier model and the set of identified 478 cluster centres. For each submitted ASAP workflow, the 479 resource provisioning algorithms probe the KB to iden- 480 tify the cluster closest (most similar) to this workflow, 481 thereby assigning it to an appropriately sized VM with 482 


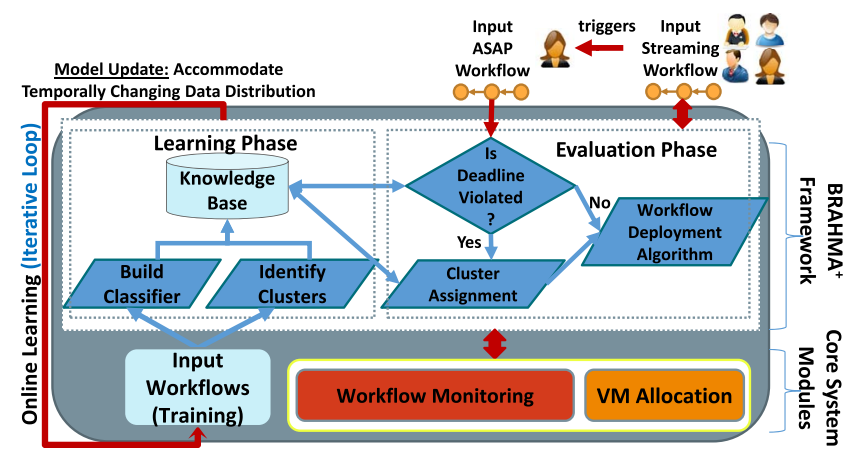

Fig. 4. Overview of the BRAHMA ${ }^{+}$Framework.

the aim to meet its deadline constraints. The KB thus serves as the decision making body for the entire framework, and hence, is kept up-to-date with all the changes resulting from the online learning process.

- Workflow Monitoring: keeps track of the progress for each component $C_{k j}$ of a workflow $W_{j}$. More specifically, it continuously probes the VMs and the workflow components to monitor the number of components running on a particular VM and the time remaining for the component to finish execution respectively. As will be explained later in Section V, the monitoring capability plays a central role in the design of the more involved pro-active and hybrid resource provisioning algorithms for streaming and ASAP workflows respectively.

- VM Allocation: facilitates on-demand creation of new VM instances based on a specific VM template from the pool of VMs $\mathcal{V}$. The core function of this module is to perform VM allocations based on the events triggered by the workflow monitoring module and the information retrieved from the KB. VM allocations broadly happen in two ways: (1) VMs are reserved in the beginning and remain fixed throughout; (2) VM reservations happen dynamically and their specifications are adapted based on the submitted workflow resource requirements.

\section{A. Learning Phase}

${ }_{508}$ BRAHMA $^{+}$(Fig. 4), operates in two phases. Firstly, in the 509 learning phase, $\mathrm{BRAHMA}^{+}$takes as input workflow requests 510 submitted to the cloud environment, which serves as its train511 ing data. To facilitate robustness and generalizability of the 512 learned models, BRAHMA $^{+}$keeps on updating its models 513 incrementally to ensure modelling a proper mix of workflows 514 with varying number of components, component types etc. ${ }_{515}$ Each workflow $W_{j}$, possesses resource usage statistics (in $516 \mathrm{MI})$ for each of its constituent component $\forall k, C_{k j}$, while also 517 containing information about its deadline status (i.e., was the 518 deadline violated or met). ${ }^{1}$ The first task of BRAHMA $^{+}$'s 519 learning phase is that of building a classifier. Here, we use 520 the classification module (described earlier in this section) to 521 analyse the generated training data and learn a classifier model ${ }_{522} \mathrm{CMM}$, based on the resource requirements and request patterns, 523 for answering the binary question: whether the deadline of an ${ }_{524}$ ASAP workflow is met or violated?

\footnotetext{
${ }^{1}$ The workflow generation process is described in detail in Section VI.
}

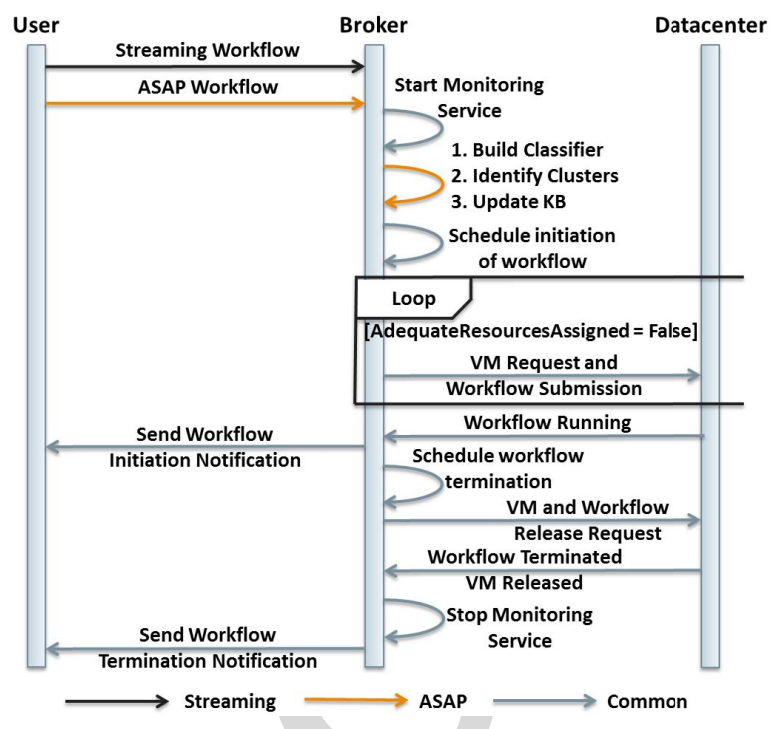

Fig. 5. Sequence diagram portraying the execution flow of both Streaming and ASAP workflows.

BRAHMA $^{+}$independently clusters similar workflows 525 (based on the resource requirements and request patterns of its 526 constituent components) from the training data to form seman- ${ }_{527}$ tically meaningful groups or clusters. As explained above, the ${ }_{528}$ (online) clustering module allows analysis of the workflow 529 behavior at a finer level of granularity, facilitating appropriate 530 resource provisioning decisions with the aim to avoid deadline ${ }_{531}$ violations. Eventually both the classifier model $\mathcal{C M}$ and the ${ }_{532}$ created set of clusters along with their cluster centers $C C$, are ${ }_{533}$ curated in the Knowledge Base (KB).

534

The learning process described till now lacks the capability 535 to tackle time-varying workflows. Hence, to effectively solve 536 the AWS-tSA problem where workflow resource requirements 537 vary over time, $\mathrm{BRAHMA}^{+}$employs the use of online learn- ${ }_{538}$ ing strategies. As is clear from Fig. 4, the learning phase ${ }_{539}$ does not represent a single-shot conventional machine learn- 540 ing pipeline, rather it is iterative and continuously refines the ${ }_{541}$ learned classifier model $C M$ and the identified cluster cen- ${ }_{542}$ ters $C$. More specifically, as and when $\mathrm{BRAHMA}^{+}$receives ${ }_{543}$ newer training data, it is ingested in the learning phase, the 544 models are updated, and eventually these updates are prop- 545 agated to the $\mathrm{KB}$ thereby facilitating the evaluation phase 546 to employ the most recently learned models for resource 547 provisioning.

\section{B. Evaluation Phase}

549

In the evaluation phase, new streaming requests along 550 with triggered ASAP requests are submitted to BRAHMA $^{+}{ }_{551}$ for inferring their execution behavior, resource requirements 552 and deadline status. As a first step, BRAHMA $^{+}$probes 553 the classifier model $C M$ saved in the $\mathrm{KB}$ to predict the ${ }_{554}$

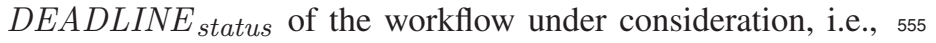
whether its deadline would be met or not. If the dead- 556 line is going to be met, then there is no need to perform ${ }_{557}$ any specialized resource scaling, as the already assigned 558 resources will be sufficient to meet the deadline-constraint 559 of the workflow. However, if a violation is predicted, we 560 


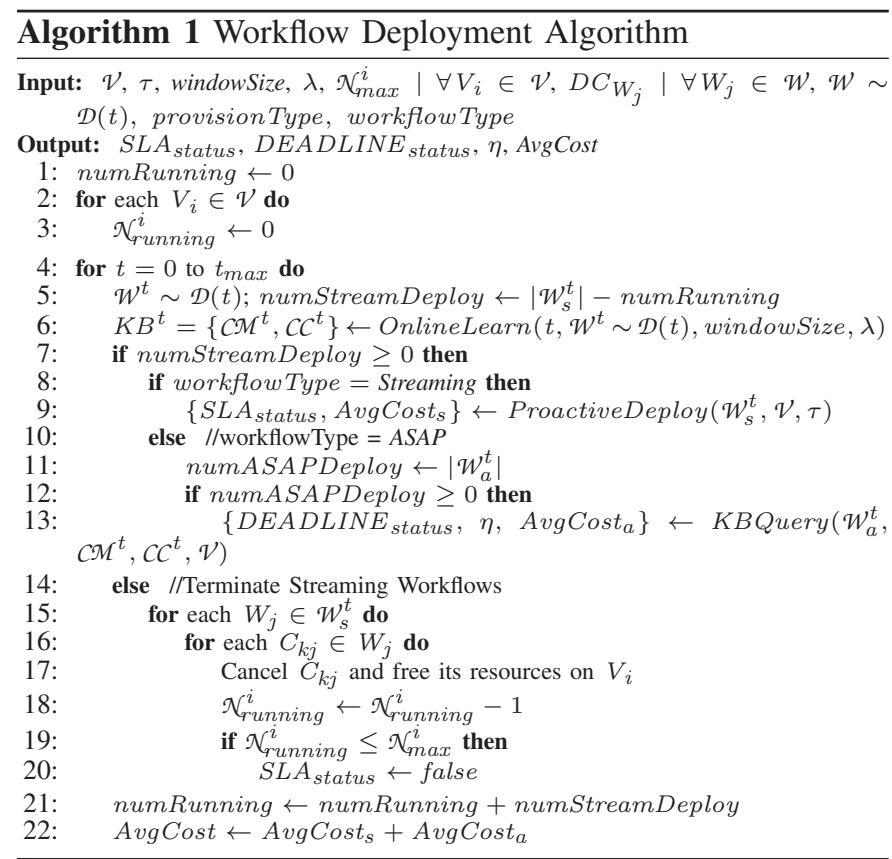

561 query the KB's cluster centers $C C$ to assign this workflow 562 to the cluster closest/most-similar to it in terms of exhibited 563 resource requirements, thereby guiding the resource provi564 sioning algorithms. While the predictions from the classifier 565 module facilitate the decision: whether to scale the resources 566 provisioned to a workflow up or down, the cluster assignments 567 from the clustering module (if the workflow was predicted to 568 violate its deadline) provide information about the resource 569 requirements of a workflow, thereby providing guidance on 570 ways to scale the resources effectively. This information is fur571 ther employed to predict the deadline status of newly incoming 572 ASAP workflows.

573 Having described the key components, two phases: learn574 ing and evaluation, and the overall execution flow of the $575 \mathrm{BRAHMA}^{+}$framework in detail, we present a sequence 576 diagram of $\mathrm{BRAHMA}^{+}$in Fig. 5. The sequence diagram 577 explains the execution flow of streaming and ASAP work578 flows, while also providing an in-depth explanation of the 579 interaction between various components of the BRAHMA $^{+}$ 580 framework using the entities in context of CloudSim [12].

\section{V. RESOURCE PROVISIONING ALGORITHMS}

582 To effectively perform resource provisioning for both time583 varying and static workflows, we present a generic workflow 584 deployment algorithm with pseudo-code listed in Algorithm 1. 585 The number of workflow requests submitted to BRAHMA ${ }^{+}$ 586 follow a time-varying distribution $\mathcal{D}(t)$. To this end, we sam587 ple requests at different discrete time-instants (line 5). If 588 the workflow under consideration is a streaming workflow, 589 we invoke the proactive algorithm (Section V-B) to scale its 590 services, with the objective of completely mitigating SLA vio591 lations and maintaining high cost-efficiency (lines 8 and 9). On 592 the other hand, for ASAP workflows the resource provisioning 593 is performed using the hybrid algorithm (Section V-C), which 594 in turn probes the curated information from the $\mathrm{KB}$ to take 595 appropriate decisions (lines 10-13). Note that as indicated in

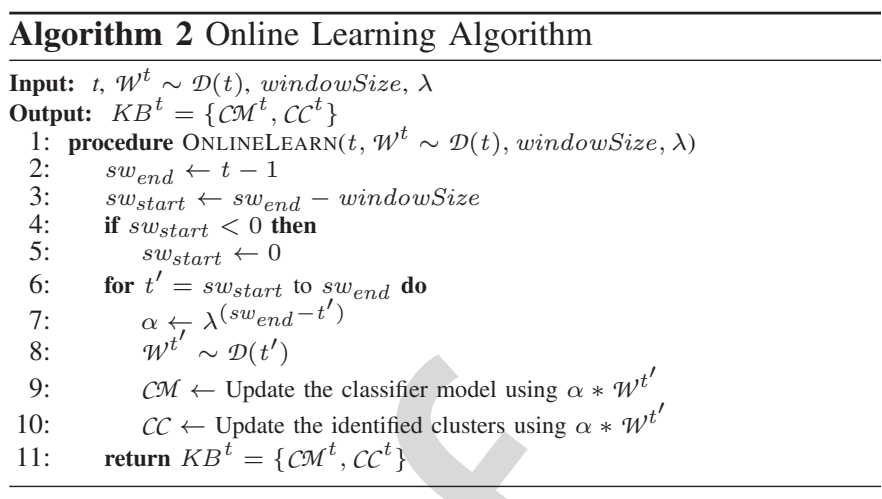

Fig. 6 streaming and ASAP workflows are provisioned on sep- 596 arate resource pools and thus, the resource provisioning deci- 597 sions made for one will not interfere with that of the other and ${ }_{598}$ vice-versa. Once workflows have been successfully executed, 599 the resources allocated to them are freed and corresponding 600 bookkeeping information is updated (lines 14-20).

As discussed previously, the resource requirements exhib- 602 ited by the submitted workflows may change over time. Since 603 the $\mathrm{KB}$ acts as an important decision making unit of the 604 workflow deployment algorithm, the information curated here 605 should be consistent and up-to-date with the latest monitored 606 workflow requirements and older (stale) information about 607 now-defunct workflow requirements should in time be phased 608 out. This is achieved using online learning (line 6), where 609 newly arriving workflows are used to update the classifier 610 model and identified clusters. Specifically, we incorporate the 611 use of a sliding window based approach, which is described 612 in the subsequent sub-section.

Note that the workflow deployment algorithm is capable 614 of handling both time-varying and static workflows. With the 615 value of windowSize $=0$, there is no window constructed 616 and the algorithm works for static workflows, while on the 617 other hand, any non-zero value of the windowSize enables 618 the algorithm to work for time-varying workflows.

619

\section{A. Online Learning Algorithm}

620

Algorithm 2 presents the pseudo-code for the online learn- 621 ing algorithm. To keep the KB up-to-date with the changing 622 workflow resource requirement patterns, we need to update the 623 learned mathematical models - classification model $\mathcal{C M}$ and 624 identified cluster centers $C C$. Therefore, the models need to be ${ }_{625}$ updated in an online manner. Since the changes in data can 626 be large, updating models for every new incoming request is 627 highly inefficient and impractical. To this end, we use a sliding 628 window based approach to handle all the updates. For every 629 newly arriving request at time $t$, a window $s w\left[s w_{\text {start }}, s w_{\text {end }}\right], 630$ where $s w_{\text {end }}=t-1$ and $s w_{\text {start }}=$ swend - windowSize, 631 is constructed (lines 2-5). The intuition is that the window 632 captures resource requirements exhibited by workflows that 633 are temporally close to the newly incoming workflow. Later, 634 the learned models $\mathcal{C M}$ and $C C$ are updated using the work- 635 flows pertaining to the constructed sliding window $S w$ and the 636 updates are translated to the KB (lines 6-11). ${ }_{637}$

To effectively incorporate new workflow resource require- 638 ments and simultaneously phase out defunct requirements we 639 


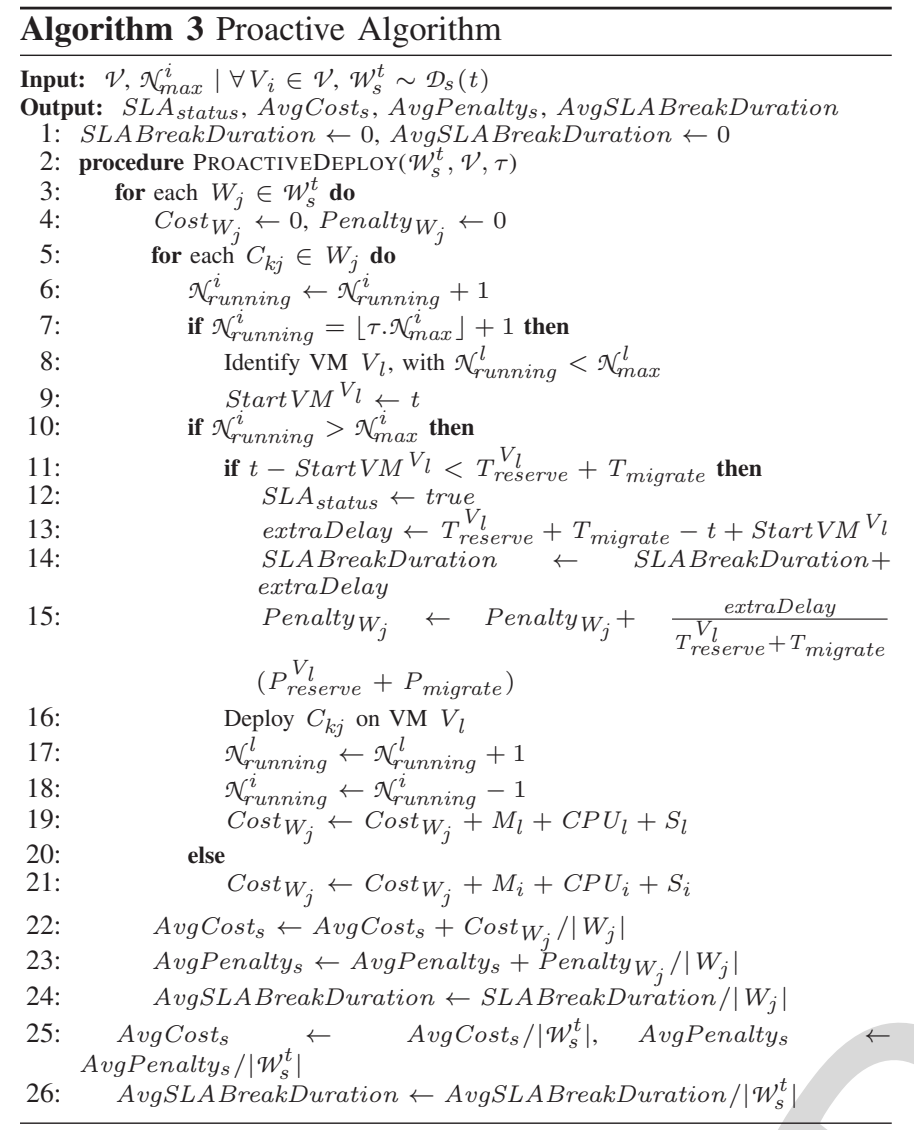

640 incorporate the use of information decay. Workflows that are 641 temporally farther from the newly incoming workflows would 642 have relatively less contribution towards learning their resource 643 requirements when compared to the workflows that are tempo644 rally closer [20]. To model this effect, we use $\lambda(<=1)$ as the 645 rate of information decay over time. More specifically, given 646 a sliding window $s w$, the contribution of workflows pertaining 647 to a time-instant $t^{\prime}$ is scaled using $\alpha=\lambda^{\left(s w_{\text {end }}-t^{\prime}\right)}$ (line 7). ${ }_{648}$ Later $\alpha$ is used to weigh the relative importance of $\mathcal{W}^{t^{\prime}}$ for 649 updates to $C \mathcal{M}$ and $C \mathcal{C}$ (lines 9-10).

\section{B. Proactive Algorithm}

651 Algorithm 3 describes the pseudo-code for the proactive 652 algorithm. In this algorithm, the SLA monitoring module 653 continuously monitors the number of service components ${ }_{654} \mathcal{N}_{\text {running }}^{i}$ and checks how far this is from the maximum per655 missible limit $\mathcal{N}_{\text {max }}^{i}$, for each VM $V_{i} \in \mathcal{V}$ (lines 6-9). 656 The proactive algorithm incorporates the use of a param657 eter $\tau$, which enables triggering of new VM reservations 658 (line 8) for service components running on a $\mathrm{VM} V_{i}$ once ${ }_{659} \mathcal{N}_{\text {running }}^{i}=\left\lfloor\tau \mathcal{N}_{\text {max }}^{i}\right\rfloor+1$ (line 7). By using the parameter ${ }_{660} \tau$, a VM is proactively started, which when ready accepts the 661 new requests for this session. More specifically, the parameter ${ }_{662} \tau$ facilitates the reservation of a new VM $V_{l}$ and the migra663 tion of service components from $V_{i}$ to $V_{l}$, while there is still ${ }_{664}$ room for more components to be executed on VM $V_{i}$ without 665 breaking the SLAs.

666 Note that since we preach maximum resource utilization, 667 although new VM reservations are triggered once the above

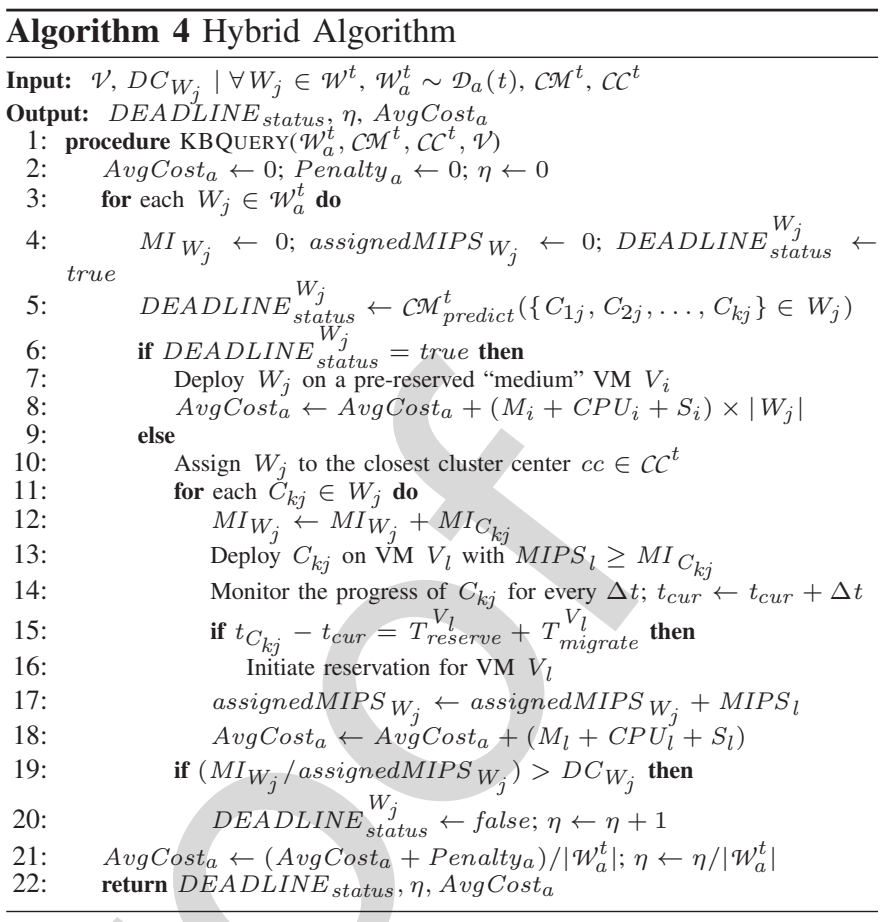

condition is met, the service components are migrated only 668 after the VMs currently running them are utilized to their max- 669 imum capacity, i.e., once for a VM $V_{i} \mathcal{N}_{\text {running }}^{i}=\mathcal{N}_{\text {max }}^{i} \cdot{ }_{670}$ Thus, once $V_{i}$ is fully utilized (line 10), the workflow com- 671 ponents are migrated to the newly reserved $\mathrm{VM} V_{l}$, and the 672 corresponding costs are updated accordingly (lines 16-19). 673

Next, we describe the effect of new VM reservations and 674 component migrations on workflow SLAs (lines 11-15). The 675 SLAs of all the components remain violated for the time 676 required to reserve new VMs and the time required to migrate 677 them from one VM to another, discounting the time dura- 678 tion corresponding to the start of the reservation process and 679 the time instant at which the SLA actually got violated. 680 Mathematically, SLABreakDuration ${ }^{C_{k j}}=$ extraDelay $=681$ $T_{\text {reserve }}^{V_{l}}+T_{\text {migrate }}-t+\operatorname{Start} V M^{V_{l}}$ (line 13 ); $\forall W_{j} \in{ }_{682}$ $\mathcal{W}_{s}^{t}, \forall C_{k j} \in W_{j}$ and $\forall V_{l} \in \mathcal{V}$. Thus, with a careful selection 683 of $\tau, T_{\text {reserve }}+T_{\text {migrate }}$ would get subsumed by the dif- 684 ference in time at which the SLAs actually got violated and 685 the time at which the reservation process was triggered. This 686 will enable SLAs to be always met while the waiting time on 687 VMs that need to be started will also be 0. Additionally, a 688 penalty proportional to the duration for which the SLAs were 689 violated is added to the costs (line 15), on top of the usual 690 VM utilization costs.

691

The proactive algorithm prevents SLA violations by closely 692 monitoring the behavior of service components. If the param- 693 eter $\tau$ is too low, additional VMs will be reserved rapidly 694 which will in turn drive up the cost. Likewise, if $\tau$ is too 695 high, new deployments will be queued until a new VM is 696 instantiated.

\section{Hybrid Algorithm}

698

Algorithm 4 presents the pseudo-code for the hybrid algo- 699 rithm. It incorporates the use of the curated information 700 from the $\mathrm{KB}$ updated and constructed by the online learning 701 


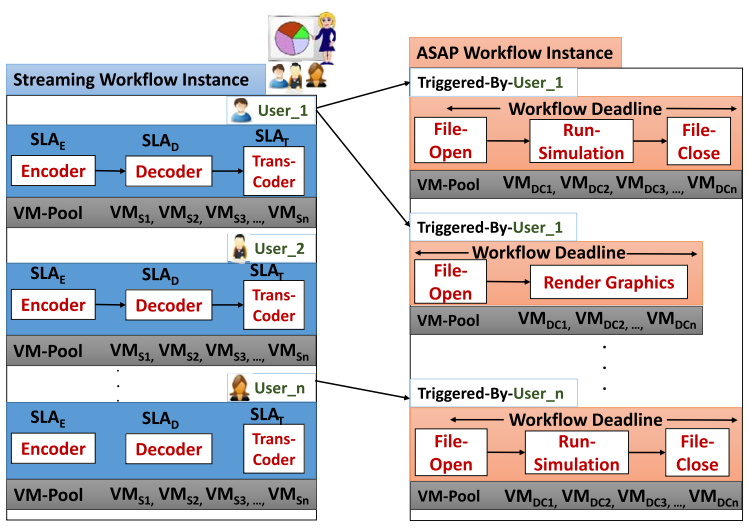

Fig. 6. Streaming workflows spawning ASAP workflows.

702 algorithm (Algorithm 2) of BRAHMA ${ }^{+}$. As a first step, 703 the hybrid algorithm invokes the classification model $\mathrm{CM}^{t}$ 704 stored in the $\mathrm{KB}$, to predict the DEADLINE status $_{\text {of each }}$ 705 ASAP workflow (line 5). The workflows with the predicted 706 DEADLINE $E_{\text {status }}=$ true do not need any specialized scal707 ing and thus, they are assigned to a "medium" (detailed in 708 Section VI) VM (lines 6-8). For workflows whose deadlines 709 are predicted to be violated, we query the identified cluster 710 centers $C C^{t}$ stored in the $\mathrm{KB}$ and try to identify the clus711 ter, which possesses workflows with the most similar resource 712 requirement patterns (line 10). Next, with this derived infor713 mation, the workflow components are assigned appropriate 714 resources accordingly (lines 11-22).

715 Specifically, each service component $C_{k j} \in W_{j}$ is assigned 716 to a VM $V_{l}$ that is large enough to honor the component 717 resource requirements (line 13). A notable limitation of the 718 hybrid algorithm is that, the resources are not pre-reserved, 719 and hence, it is prone to suffer from various penalties incurred 720 owing to new VM reservations and migration of workflow 721 components from one VM to another. To mitigate or mini722 mize these penalties, the hybrid algorithm incorporates the use 723 of monitoring (similar to the proactive algorithm described in ${ }_{724}$ Section V-B) to continuously track the progress of an execut725 ing component. More specifically, for every clock tick $\Delta t$, 726 a monitor event tracks the execution status of a currently 727 running component $C_{k j}$ (line 14), and as soon as the time 728 left for its execution to complete, crosses the VM reserva729 tion and migration time $T_{\text {reserve }}^{V_{l}}+T_{\text {migrate }}^{V_{l}}$ (line 15), a new ${ }_{730} \mathrm{VM}$ reservation is triggered. This enables timely reservation ${ }_{731}$ of new VMs and migration of components, thereby mitigating 732 the incurred penalties completely (lines 14-16).

${ }_{734}$ A. Media Workflows

735 The media workflows (Fig. 6) used in this study are inspired 736 by the EMD project ${ }^{2}$ and the online meeting room use-case

\footnotetext{
${ }^{2}$ The EMD project is an imec funded project aimed at design and development of an elastic platform for media distribution in the context of online collaborative services. The research done in this article is inspired by the diverse workflow types observed in EMD, which in addition to presenting a real-world scenario also possess high affinity to the use-case of online collaborative meeting discussed in this article. Additional details about EMD are available at: https://www.imec-int.com/en/what-we-offer/research-portfolio/emd.
}

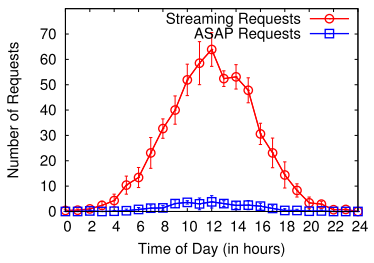

(a)

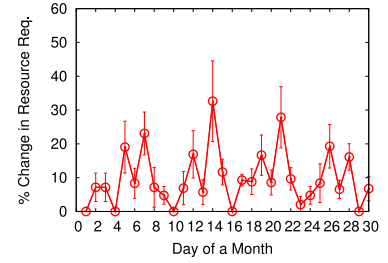

(b)
Fig. 7. Variation in the (a) number of Streaming and ASAP worfklow requests based on the time of day, and (b) change of workflow resource requirements with day of the month.

discussed in Section I. Each user participating in the meet- ${ }_{737}$ ing represents an instance of a streaming workflow, and can 738 additionally trigger multiple ASAP workflows. 739

Components corresponding to streaming workflows possess 740 an SLA, which, if not met, may cause unwanted side-effects. 741 Staying with the use case at hand this could cause A/V 742 synchronization issues, stuttering, etc. Each ASAP work- ${ }^{743}$ flow possesses a deadline-constraint, which can be either met 744 or violated, and if violated causes simulation results to be 745 delayed, or high-quality graphics not to be rendered properly. 746 Additionally, the resource requirements exhibited by individ- 747 ual components of the submitted workflows vary over time. 748 Note that even though much more elaborate workflows exist, 749 these particular workflows have been chosen to showcase the 750 strength of $\mathrm{BRAHMA}^{+}$and the presented algorithms in an 751 easy-to-grasp manner. Moreover, BRAHMA ${ }^{+}$and its associ- 752 ated algorithms are able to work with generic workflows, and 753 are not constrained by the above assumptions.

\section{B. Evaluation Scenario}

As shown in Fig. 7a, 200 user requests for streaming work- 756 flows are generated following a normal distribution, with the 757 time 12 noon set as mean and 3.5 hours as standard deviation. 758 At every time instant, each user further possesses a 5\% chance 759 of triggering an ASAP workflow. This graph portrays that the 760 number of requests for both streaming and ASAP workflows 761 will vary in between the start of the day up to the end of the 762 day: high load during office hours and negligible load during 763 evening and night time.

Each streaming workflow possesses 3 components, that 765 execute continuously during the course of the meeting. 766 ASAP workflows, on the other hand, can be of varying 767 characteristics: number of components, resource require- 768 ments etc. To this end, a request generator module gen- 769 erates a variety of 3,4 , and 5 component workflows 770 (obtained from our industrial partners in the EMD [4] 771 project.), where the resource requirements of each compo- 772 nent correspond to the templates as shown in Table II. For 773 example, the file-open $\rightarrow$ run-simulation $\rightarrow$ file-close workflow 774 corresponds to the $<$ low $\rightarrow$ high/very-high $\rightarrow$ low $>$ template. A 775 map-reduce job could correspond to the $<$ low $\rightarrow$ high/very- 776 high $\rightarrow$ low $\rightarrow$ high/very-high $\rightarrow$ low $>$ template. The deadline- 777 constraint of each ASAP workflow is estimated using the 778 expected resource requirement of a component. Specifically, 779 the deadline-constraint, represented in terms of MI require- 780 ments, of a workflow $W_{j}$ possessing k components is calcu- 781 lated as $\mathrm{k}$ times the expected component resource requirement. 782 
TABLE II

RESOURCE REQUIREMENT TEMPLATES

\begin{tabular}{|c||c|c|c|c|c|}
\hline Template Name & Very-Low & Low & Medium & High & Very-High \\
\hline $\begin{array}{c}\text { Resource requirement } \\
\text { range (MI) }\end{array}$ & $100-300$ & $250-550$ & $500-800$ & $750-1050$ & $1000-1200$ \\
\hline
\end{tabular}

${ }_{783}$ To better evaluate BRAHMA $^{+}$in real-world scenarios, in 784 addition to workflows where resource requirements of compo785 nents are assumed to be static, we also conduct experiments 786 with time-varying workflows: where resource requirements of 787 the constituent service components vary over time. We simu788 late this temporal change using the hypothesis that the resource 789 requirements of service components may undergo a change on 790 a daily basis, with the change being small (of the order of 5\%) 791 during the weekdays and large (of the order of 25\%) during 792 the weekends. Fig. $7 \mathrm{~b}$ portrays this behaviour. The days repre793 sented as numbers start from Monday, thus, Day 1 represents 794 Monday, Day 12 represents Friday, and so on. As can be seen, 795 the highest change occurs on Friday, portraying transitioning 796 of resource request patterns from weekdays to weekends, and 797 on Sunday, which presents the reverse effect, i.e., the change 798 from weekends to weekdays.

799 For each user request a new instance of the workflow $W_{j}$ 800 is created and the constituent service components $C_{k j}, \forall k \mid$ ${ }_{801} C_{k j} \in W_{j}$ are provisioned on different VMs $V_{i}$, available 802 from the VM pool $\mathcal{V}$ (the choice of which VM and how 803 this VM pool grows / shrinks is driven differently depending 804 on the choice of algorithm). To deploy VMs in the resource 805 pools, eight types of VM images were defined as detailed 806 in Table III. The costs for the VM templates used were 807 parametrized based on the Amazon EC2 image c3.8xlarge [1], 808 with a monthly price of $\$ 1.680$ to provide 32 vCPUs (17476 809 MIPS [2]), 60 GB of RAM and $2 * 320$ GB of storage. This 810 cost was divided equally between secondary-storage, main811 memory and CPU, and the converted unit prices (per MB/hour 812 and MIPS/hour [3]) were used to calculate the costs for the $813 \mathrm{VM}$ templates used in this article. As mentioned in Section V, 814 the time required to reserve new VMs differs significantly 815 from the time required to migrate one component from an 816 existing VM to another. To this end, we define two vari817 ables, $T_{\text {reserve }}^{V_{i}}$ and $T_{\text {migrate }}$, that determine the duration for 818 instantiating new VMs and the duration for migrating compo819 nents from an existing VM instance to another respectively. 820 For the simulations, the values of $\left(T_{\text {reserve }}^{V_{i}}\right)$ and $\left(T_{\text {migrate }}\right)$ 821 were defined as uniform distributions between [40s,55s] and $822[0.5 \mathrm{~s}, 2 \mathrm{~s}]$ respectively using recommendations provided in [27] 823 and [40]. Additionally, the specific values were extrapolated 824 to correspond to the VM images used in this study. All the 825 parameters mentioned above are not constrained to the stated 826 fixed values, and can be tuned as needed.

\section{${ }_{827}$ C. Evaluation Metrics}

- Efficacy: We adopt SLA status (Definition 5), average SLA violation duration (Definition 6), and deadline status (Definition 8) [8], [9] to evaluate the quality of the discussed methods.

- Cost: We use the VM cost (Definition 9), and penalty (Definition 10) [8], [9] to measure the incurred cost.
TABLE III

PARAMETERIZED VM TEMPLATES

\begin{tabular}{|c|c|c|c|c|}
\hline Template & CPU & RAM & Storage & Hourly Cost \\
\hline \hline Template $_{01}$ & $150 \mathrm{MIPS}$ & $4 \mathrm{~GB}$ & $128 \mathrm{~GB}$ & $\$ 0.154$ \\
\hline Template $_{02}$ & $300 \mathrm{MIPS}$ & $8 \mathrm{~GB}$ & $256 \mathrm{~GB}$ & $\$ 0.308$ \\
\hline Template $_{03}$ & $450 \mathrm{MIPS}$ & $12 \mathrm{~GB}$ & $384 \mathrm{~GB}$ & $\$ 0.462$ \\
\hline Template $_{04}$ & $600 \mathrm{MIPS}$ & $16 \mathrm{~GB}$ & $512 \mathrm{~GB}$ & $\$ 0.616$ \\
\hline Template $_{05}$ & $750 \mathrm{MIPS}$ & $20 \mathrm{~GB}$ & $640 \mathrm{~GB}$ & $\$ 0.77$ \\
\hline Template $_{06}$ & $900 \mathrm{MIPS}$ & $24 \mathrm{~GB}$ & $768 \mathrm{~GB}$ & $\$ 0.924$ \\
\hline Template $_{07}$ & $1050 \mathrm{MIPS}$ & $28 \mathrm{~GB}$ & $896 \mathrm{~GB}$ & $\$ 1.078$ \\
\hline Template $_{08}$ & $1200 \mathrm{MIPS}$ & $32 \mathrm{~GB}$ & $1024 \mathrm{~GB}$ & $\$ 1.232$ \\
\hline
\end{tabular}

\section{Methods Benchmarked}

834

We compare the cost and efficacy of the Proactive and 835 Hybrid algorithms, proposed under $\mathrm{BRAHMA}^{+}$, against a 836 number of carefully designed baselines and heuristics.

For streaming workflows, we employ the use of passive 838 and reactive algorithms [9] for comparison. Under the pas- 839 sive algorithm, all resources are reserved in the beginning 840 of the application session, and do not undergo any change ${ }_{841}$ even if their capacity is reached. On the other hand, the reac- ${ }_{842}$ tive algorithm allows new resources to be reserved once the ${ }_{843}$ pre-reserved resources reach their capacity.

844

For ASAP workflows, we use the baseline and advanced 845 algorithms [8] as benchmarks. The baseline algorithm is 846 similar to the passive algorithm in design: it reserves all 847 the resources at the beginning of the application session. 848 Every incoming ASAP workflow is assigned to a pre-reserved 849 "medium" (Template04 in Table III) sized VM. An intuitive 850 approach to define the MIPS of a medium-sized VM is using 851 the expected MI requirement of a workflow component. The 852 reason being that in expectation, this VM would be able to 853 meet the deadline constraints of half of the ASAP work- ${ }^{854}$ flows. The advanced algorithm on the other hand allows new 855 resources to be provisioned when the pre-reserved VM is not 856 sufficient.

857

Lastly, for time-varying workflows, we use the non time 858 window enabled versions of the proposed algorithms as bench- 859 marks. These algorithms ignore the capability of BRAHMA ${ }^{+}{ }_{860}$ to adapt to the changing resource requirements of workflows. 861 More specifically, after the initial learned models are pop- 862 ulated in the $\mathrm{KB}$, they are not updated as the workflow 863 requirements change over time, and the benchmarks work with 864 this non-updated copy of the KB instead.

\section{EVAluation RESUlts}

866

All simulations were performed using the CloudSim sim- ${ }^{867}$ ulator [12] and its extensions ${ }^{3}$ proposed in this article, on ${ }_{868}$ an Intel(R) Core i5 4-core machine with $1.7 \mathrm{GHz} \mathrm{CPU}$ and ${ }_{869}$ 8 GB RAM running Linux Ubuntu 16.04. We use the publicly 870 available implementations of the classification and clustering 871 models from the WEKA [18] data mining software. Results are 872 averaged over 10 simulation runs. Note that all the parameter 873 values/ranges recommended in the following section(s) are a 874 result of fine-tuning based on the workload and experimental 875 setup employed in this study. The recommended values/ranges 876 are thus, not generic, and subject to change on new workloads. 877

\footnotetext{
${ }^{3}$ The code (along with a description of the CloudSim extensions) will be open sourced to the research community via GitHub.
} 


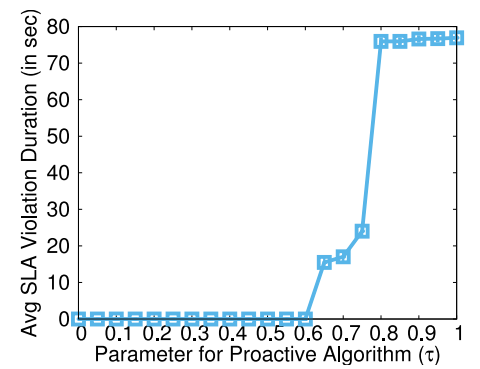

(a)

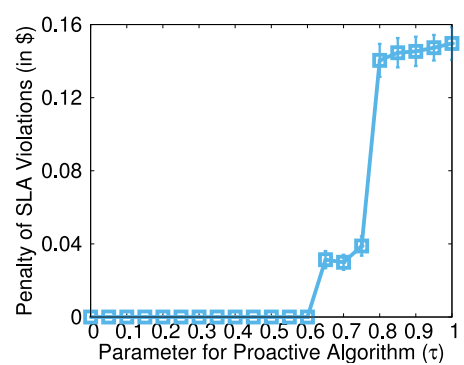

(b)

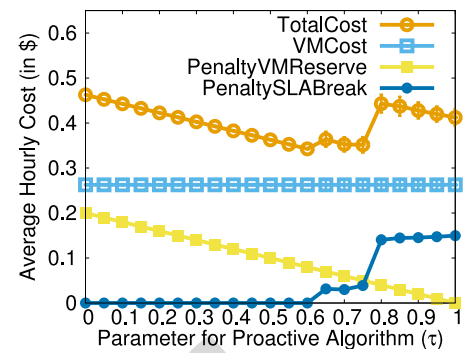

(c)

Fig. 8. Variation in the (a) average SLA violation duration, (b) average penalty, and (c) average cost as a function of $\tau$ for the proactive algorithm.

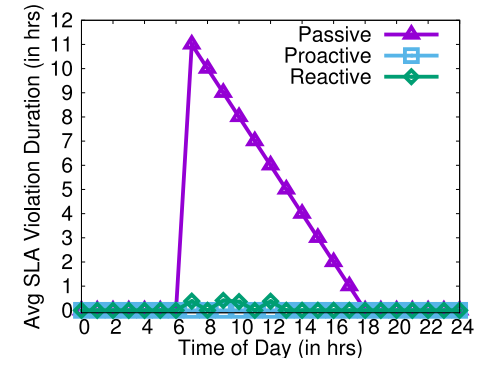

(a)

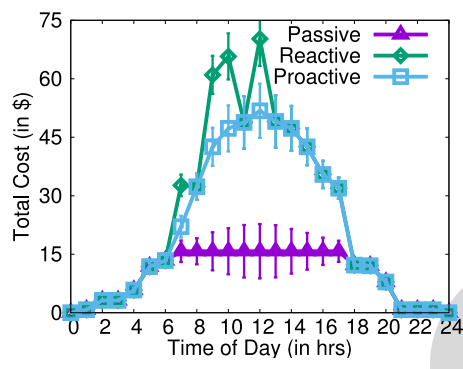

(b)

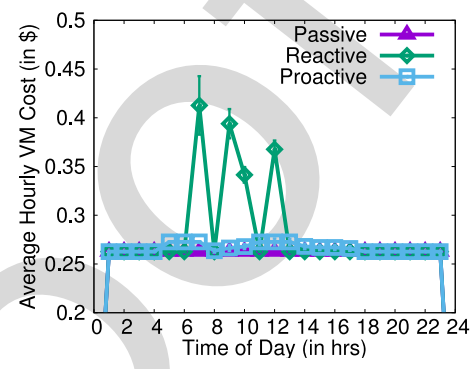

(c)

Fig. 9. A comparison of the (a) average SLA violation duration, (b) total cost, and (c) average cost as a function of the time of day for the passive, reactive and proactive algorithm. The reported costs are parametrized using VM templates stated in Table III.

\section{A. Streaming Workflows}

879 The proactive algorithm possesses a parameter $\tau$ that con880 trols triggering of new VM reservations. As mentioned in ${ }_{881}$ Sections V and VI, once $\mathcal{N}_{\text {running }}^{i}>\tau \mathcal{N}_{\text {max }}^{i}$, a new VM 882 reservation is triggered by the resource provisioning modules. 883 Note that as stated in Section V-B and [9], $\tau$ should neither be 884 too high nor too low. The former will lead to large number of 885 SLA violations as workflows would be queued waiting for a 886 new VM to be instantiated, while the latter would lead to high 887 cost, which might be prohibitive. Thus, as a general guideline ${ }_{888} \tau$ should use a moderate value, viz. $0.25 \leq \tau \leq 0.75$, for opti889 mizing the trade-off. Next, we analyze the impact of $\tau$ on the 890 proactive algorithm in the context of our experimental setup. 891 Fig. 8a shows that the SLAs of the components are met when $892 \tau \leq 0.6$, beyond which the average SLA violation duration 893 starts increasing. $0 \leq \tau \leq 0.6$ serves as a good range with 894 respect to minimizing the SLA violation duration.

895 The average penalty incurred during the time when SLAs 896 are violated is shown in Fig. 8b. Since the penalty is incurred 897 due to SLA violations, it is not surprising that the slope of 898 the curve in Fig. $8 \mathrm{~b}$ is highly similar to that of Fig. 8a. Thus, 899 even with respect to minimizing the average penalty, parameter 900 values in the range $0 \leq \tau \leq 0.6$ are considered to be optimal. 901 Fig. 8c presents the average cost incurred with varying $\tau$. 902 The average VM cost (Eq. 4) is almost constant with the vari903 ation in $\tau$. It is evident from Fig. 8c that the penalty incurred 904 due to proactive reservations of VMs decreases linearly with 905 the increase in parameter $\tau$. More specifically, this penalty 906 assumes its maximum value when $\tau=0$ and its mini907 mum value when $\tau=1$. The total cost is the sum of the ${ }_{908} \mathrm{VM}$ cost and the two penalties discussed above. It is evi909 dent that the total cost first linearly decreases till $\tau=0.6$, 910 becomes almost constant till $\tau=0.75$ and then starts to increase with increasing $\tau$. Thus, with respect to minimizing 911 the total cost, $0.6 \leq \tau \leq 0.75$ serves as the optimal parameter ${ }_{912}$ range.

In sum, the value $\tau=0.6$ serves as the best possible trade- 914 off for minimizing the costs while also keeping the SLAs of 915 the components in line. Note that the proactive algorithm will 916 use $\tau=0.6$ for all of the following analyses. 917

Fig. 9a shows a comparison of variation in the SLA vio- 918 lation duration depending on the time of day for the three 919 proposed algorithms. The SLA violation duration under the 920 proactive algorithm is always 0 , as the SLAs are always met, ${ }^{21}$ while for the reactive algorithm it is jittery characterized by 922 spikes where SLAs get violated. The SLA violation duration ${ }_{923}$ under the passive algorithm increases suddenly to its maxi- ${ }_{924}$ mum value and then linearly decreases till it becomes 0 . The 925 reason for this phenomenon is as follows: the SLA first gets 926 violated at 7 in the morning and remains violated until 6 in ${ }_{927}$ the evening. Thus, SLAs for the components arriving at 7 AM 928 remain violated for 11 hours, those arriving at 8 AM remain ${ }_{929}$ violated for 10 hours and so on.

Fig. 9c presents a comparison of the variation in the aver- 931 age cost (per component) with the time of day for the three 932 proposed algorithms. It is evident that the average cost of 933 the algorithms are almost similar (except for reactive, which 934 is characterized by spikes at some instances) at majority of ${ }_{935}$ the time instances. Note that the costs portrayed in Fig. 9c 936 also include the penalties (as explained in Section V) incurred ${ }_{937}$ by the resource provisioning algorithms. Moreover, since no 938 penalties are incurred by the passive algorithm, the cost ${ }_{939}$ reported equals the VM utilization cost. At certain instances, 940 the average cost of the reactive algorithm is the highest, which 941 is the result of the penalties incurred due to the VM reserva- 942 tion process starting only after the SLAs are violated. Since the ${ }_{943}$ 


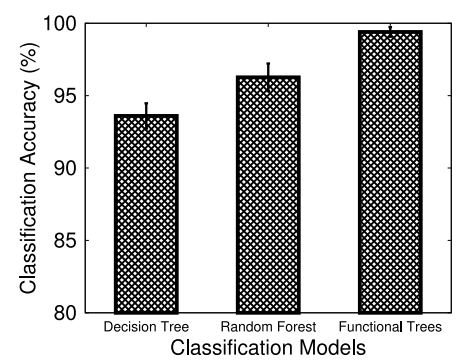

(a) Classifier Performance

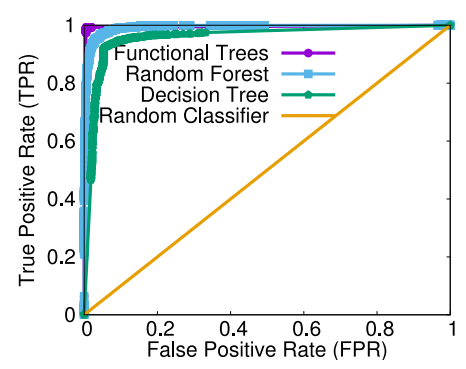

(b) ROC curve analysis

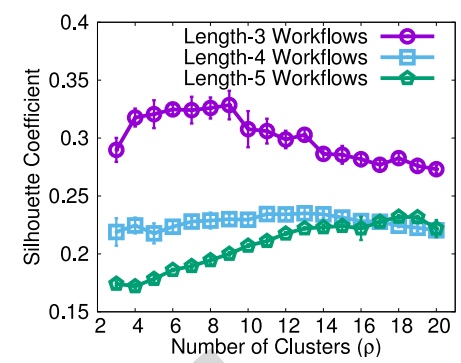

(c) Impact of $\rho$ on cluster coherency

Fig. 10. Evaluations for the learning phase of the $\mathrm{BRAHMA}^{+}$framework

944 proactive algorithm triggers the new VM reservation process 945 prior to detecting violation in the SLAs, the penalties incurred 946 for this algorithm are significantly lower when compared to 947 that of the reactive algorithm. The only penalty incurred 948 on the proactive algorithm is due to the pre-reservation 949 of VMs, which is optimized for $\tau=0.6$ as discussed 950 above.

\section{B. ASAP Workflows}

${ }_{952}$ To simulate previously executed workflows and train the 953 learning phase of $\mathrm{BRAHMA}^{+}$, we use a request genera954 tor to generate 6000 ASAP workflow requests of varying 955 ( 3 , 4, and 5) lengths. Using the deadline estimation discussed 956 in Section VI, each ASAP workflow is then assigned a class 957 label, i.e., whether the deadline of this workflow was violated 958 or met. If the total MI requirements of an ASAP workflow is 959 greater than the estimated deadline-constraint (in MI), then its 960 deadline is marked to be violated.

961 We use the decision tree (J48 algorithm) [33], random for962 est [11] and functional tree [15] classification methods. A 963 grid-search is performed to choose the optimal set of internal 964 classifier parameters. The confidence factor used for pruning 965 the decision tree is set to 0.25 , while the minimum number of 966 instances per leaf node of the tree is set to 2 . The random for967 est classifier is built using 50 trees, and each tree is constructed 968 using 3 random features from the data. Lastly for functional 969 trees, the minimum number of instances in a node for it to 970 be considered for splitting is set to 15 , while the number of 971 boosting iterations is set to 15 . The reader is referred to [18], 972 for an in-depth understanding of these parameter terminologies 973 and their description.

974 Fig. 10a portrays the classification accuracy using 10-fold 975 cross validation, with functional tree possessing the highest 976 accuracy $(\approx 99 \%)$ while decision tree possesses the least $977(\approx 94 \%)$. Nevertheless, using any of the three classifiers, ${ }_{978}$ BRAHMA $^{+}$possesses a reasonably high classification accu979 racy. Note that our contribution is not limited to the three 980 classifiers used to portray these results, rather is based on the ${ }_{981} \mathrm{BRAHMA}^{+}$framework which suggests the use of classifica982 tion as a method in general. We also construct the receiver 983 operating characteristic (ROC) curve for the classifiers, that ${ }_{984}$ plots true positive rate (TPR) against the false positive rate 985 (FPR). Classifiers whose ROC curves approach the top-left 986 corner of the plot are considered to be good. The line $y=x$, 987 for a binary classification task, represents a random-classifier.
Fig. 10b clearly shows that all three evaluated classifiers 988 are significantly better when compared to a random method, 989 and approach the top-left corner of the plot. Moreover, both 990 functional tree and random forest possess a very good area 991 under the ROC curve (AUROC $\approx 0.99$ ).

992

We employ the use of the k-means clustering algorithm [19] 993 to cluster ASAP workflows into groups with similar resource 994 requirement patterns. Since k-means requires the number of 995 clusters to be identified as input, we employ the use of silhou- 996 ette coefficient [36]: a statistical metric for quantifying cluster 997 quality, to correctly identify the optimal number of clusters. 998 Fig. 10c plots the silhouette coefficient values for ASAP work- 999 flows of lengths 3, 4 and 5, with varying number of clusters 1000 $\rho$ from 3 to 20 . The silhouette coefficient gradually increases 1001 with the increase in $\rho$, stabilizes near a peak value, and then 1002 starts to decrease. The higher the silhouette value, the better ${ }_{1003}$ the produced clustering, thus, we choose $\rho$ as 9,11 and 18 for 1004 the length 3, 4 and 5 workflows respectively.

1005

We evaluate (Fig. 11a) the fraction of ASAP workflows 1006 whose deadline gets violated with varying deadline thresholds 1007 for the baseline algorithm. A large number of workflows, of the 1008 order of $50-60 \%$, with the worst-case being up to $78 \%$, suffer 1009 deadline violations. Moreover, only after relaxing the dead- 1010 line threshold by 40\%, each ASAP workflow is able to meet 1011 its deadline. This result portrays that naïvely assigning ASAP 1012 workflows to a "medium-sized" VM is not sufficient, and ${ }_{1013}$ hence, motivates the need for a framework like BRAHMA ${ }^{+} \cdot{ }_{1014}$

Fig. 11b presents a comparison of the baseline, advanced 1015 and hybrid algorithms in terms of the percentage of ASAP 1016 workflows whose deadline gets violated with the time of day. 1017 Since the baseline algorithm does not perform any efforts 1018 to perform intelligent resource provisioning, it suffers from 1019 a large number (up to 45\%) of deadline violations. On the 1020 other hand, the advanced and hybrid algorithms leverage the 1021 BRAHMA $^{+}$framework to perform informed resource provi- 1022 sioning, and do not suffer deadline violations for a majority of ${ }_{1023}$ the time-instances. Even when the deadlines do get violated, 1024 the percentage of violations are as low as 3-5\%. 1025

Lastly, we perform a comparison of the variation in average 1026 hourly VM costs for the proposed algorithms with the time of 1027 day. Note that this analysis includes the costs for both stream- 1028 ing and ASAP workflows as well as the penalties incurred, if ${ }_{1029}$ any. The pro-active algorithm is used with $\tau=0.6$, since the ${ }_{1030}$ SLAs are always met and there are no penalties incurred due 1031 to SLA violations. Fig. 11c shows that the baseline algorithm 1032 possesses the least cost. This is mainly due to pre-assignment ${ }_{1033}$ 


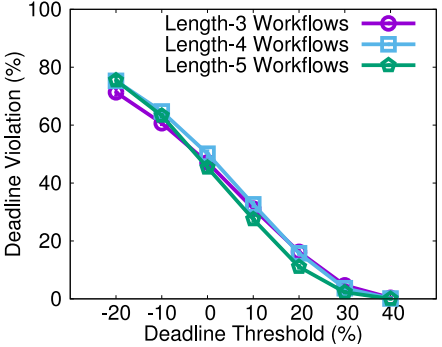

(a) Deadline Violations in Baseline

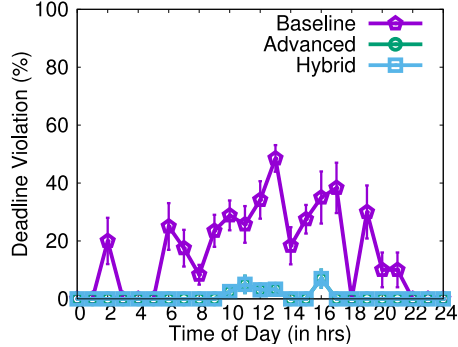

(b) ASAP Workflow Deadline Violation $(\%)$

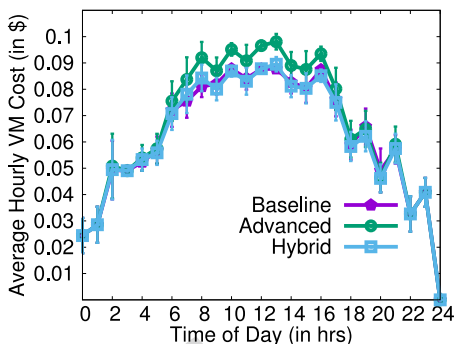

(c) Average Hourly VM Cost

Fig. 11. (a) Analysing ASAP workflow deadline violation percentage under the baseline algorithm with varying deadline thresholds. (b) A comparison of the variation in the ASAP workflow deadline violation percentage and (c) the average total cost (combining costs for streaming and ASAP workflows) versus the time of day for the baseline, advanced and hybrid algorithm.

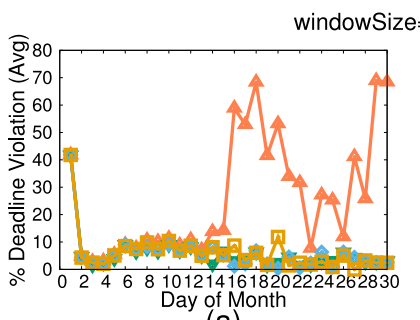

(a)

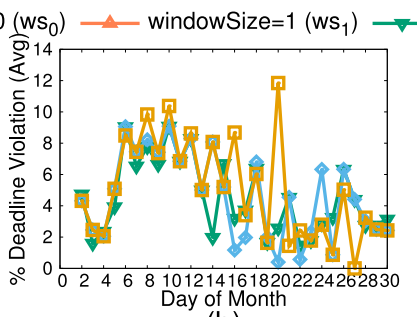

(b)

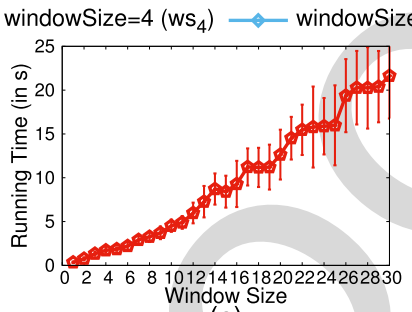

(c)

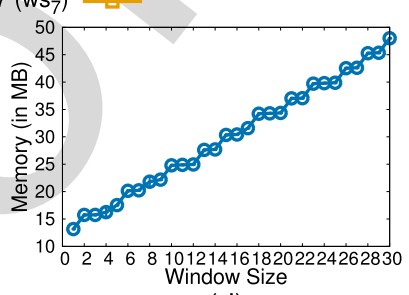

(d)

Fig. 12. (a), (b) A comparison of the variation in the average deadline violation percentage versus the day of a month for varying window sizes 0 , 1 , 4 , and 7. Rate of growth of (c) execution time and (d) memory consumption of the clustering algorithm with increase in window size from 1 to 30.

1034 of resources and the lack of new VM reservations even if $1035 \forall V_{i} \in$ pre-reserved $\mathcal{V}$, the $M I P S_{i}$ is insufficient to fulfil the 1036 requirements of a workflow $W_{j}$, which results in deadline vio1037 lations. On the other hand, the advanced algorithm possesses 1038 the highest cost, owing to penalties incurred by workflows 1039 waiting for new VM reservations and component migrations. 1040 The hybrid algorithm mitigates these penalties by using a mon1041 itoring capability similar to that of the pro-active algorithm, 1042 thereby closely mirroring the cost of the baseline algorithm 1043 and being as cost-effective.

\section{C. Time-Varying Workflows}

1045 For time-varying workflows, we generate 30 different 1046 batches of workflow requests, which are sampled daily for 1047 a period of one month. We generate 6000 ASAP workflow 1048 requests of varying lengths for each day in a month. As dis1049 cussed previously, the resource requirements (in MI) of the 1050 workflow components change over time (Fig. 7b).

1051 Conventional clustering methods that assume the underly${ }_{1052}$ ing resource request patterns to be static, are not capable ${ }_{1053}$ of capturing the behaviour exhibited by time-varying work1054 flows since their distribution of resource requirements 1055 vary over time. As discussed in Section V, to enable $1056 \mathrm{BRAHMA}^{+}$perform resource provisioning for time-varying 1057 workflows (AWS-tSA), we employ the use of sliding win1058 dows $s w\left[s w_{\text {start }}, s w_{\text {end }}\right]$ that provide an effective mechanism 1059 for updating the learned mathematical models.

1060 First, we analyse the effect of using a sliding window on 1061 the average deadline violation percentage. Fig. 12a presents the 1062 variation in average deadline violations for different window 1063 sizes. All the evaluations use the hybrid resource provision1064 ing algorithm of $\mathrm{BRAHMA}^{+}$, with the only change being the way in which the classification and clustering modules of 1065 BRAHMA $^{+}$learn the workflow behaviour. We measure the 1066 violations in workflow deadlines by varying the sliding win- 1067 dow size, where $w s_{0}, w s_{1}, w s_{4}$, and $w s_{7}$ represent approaches 1068 with window sizes $0,1,4$, and 7 respectively. The window 1069 size of 1 means that for requests generated on a day t, we 1070 will consider the day $t-1$ for performing the cluster iden- 1071 tification step; a window size of 4 means that we use the 1072 days $t-1, t-2, t-3$ and $t-4$. The procedure for any 1073 other non-zero window size follows similarly. On the other 1074 hand, a window size of 0 represents the absence of sliding 1075 windows, i.e., the conventional clustering method [8] used for 1076 workflows with static resource requirements (Section VII-B). 1077 Since the deadline violations observed under $w s_{0}$ can be 1078 as large as $70 \%$ (Fig. 12a), to better visually portray and 1079 analyse the variation of deadline violations under $w s_{1}, w s_{4}, 1080$ and $w s_{7}$, we plot Fig. 12b, which is a zoomed-in version 1081 of Fig. 12a, by ignoring the deadline violations observed 1082 under $w s_{0}$.

It is evident from Fig. 12a that the performance of $w s_{0} 1084$ degrades over time. This is because the resource require- 1085 ments of workflows change over time (Fig. 7b), however, 1086 $w s_{0}$ does not perform any effort to adapt to the chang-1087 ing resource requirements. Owing to the absence of slid- 1088 ing windows, the models are not updated and hence, the 1089 identified clusters perform worse over time, and no longer 1090 remain a representative of the resource requirements exhib- 1091 ited by the currently submitted workflows. Consequently, 1092 there is an increase in deadline violations. It is interesting 1093 to note that the same hybrid algorithm (with $w s_{0}$ ) is near- 1094 optimal (Fig. 11b shows that most of the deadlines were 1095 met) when the resource requirements of workflows were 1096 static. 
1098 Figs. 12a and 12b show that the performance of the 1099 approaches in terms of preserving the deadline constraints of 1100 time-varying workflows follows the following order: $w s_{4} \approx$ $1101 w s_{1}>w s_{7}>>w s_{0}$. A careful analysis of Fig. 12b shows 1102 that in the majority of the cases, $w s_{7}$ is worse than $w s_{1}$ 1103 and $w s_{4}$. A probable explanation of this is that $w s_{7}$ cap1104 tures too much historical information, i.e., it learns a lot of 1105 noise as well owing to the large window size. Moreover, 1106 on average $w s 4$ provides smaller deadline violations when 1107 compared to $w s_{1}$. Thus, in terms of avoiding deadline viola1108 tions, $w s_{4}$ provides a good choice for the window size in our 1109 scenarios.

1110 Having analysed the effect of window size on deadline 1111 violations, we also study its effect from a computational stand1112 point. Figs. 12c-d present the impact of variation in window 1113 size on execution time and memory consumption of the clus1114 tering module respectively. It is evident that both execution 1115 time and memory consumption increase with increase in the 1116 window size. However, as can be seen from Fig. 12c, the rate 1117 of growth of execution time is super-linear since the worst 1118 case complexity of $k$-means is super-linear. On the other hand, 1119 the rate of growth in memory-consumption is close to linear 1120 (Fig. 12d).

1121 We recommend 4 as the choice for window size as it min1122 imizes deadline violations, while being only marginally more 1123 expensive than $w s_{1}$ on computational fronts. To summarize, 1124 for the presented use-cases, $\mathrm{BRAHMA}^{+}$with its associated 1125 proactive algorithm using $\tau=0.6$, the hybrid algorithm, and 1126 the sliding window approach with window size 4 serves as 1127 the best possible trade-off for minimizing the costs while 1128 also keeping the SLAs and the deadline-constraints of the 1129 workflows in line for both static (AWS-SA) and time-varying 1130 (AWS-tSA) workflows.

\section{VIII. CONCLUSION AND FUTURE WORK}

${ }_{1132}$ In this article, we addressed the problem of Automatic 1133 Workflow resource Scaling under the combined presence of ${ }_{1134}$ Streaming and $A S A P$ workflows, called $A W S-S A$, and its time1135 varying variant called $A W S-t S A$. Consequently, we devised a 1136 holistic solution for both the problems; by coming up with a 1137 framework $B R A H M A^{+}$that curates a $\mathrm{KB}$ of learned workflow 1138 behavior(s), the proactive algorithm for streaming workflows, 1139 and the hybrid $\mathrm{KB}$ driven resource provisioning algorithm that 1140 leverage $\mathrm{BRAHMA}^{+}$for effective scaling of ASAP work1141 flows. We also portrayed the capability of $\mathrm{BRAHMA}^{+}$to 1142 adaptively learn the workflow behavior of time-varying work1143 flows, thereby facilitating online updates to the $\mathrm{KB}$ and 1144 effective resource provisioning where resource requirements 1145 change over time. Our empirical studies show that the pro1146 posed algorithms are effective and provide good cost-efficacy 1147 trade-offs. The proposed hybrid algorithm - combining learn1148 ing and monitoring, is able to restrict deadline violations to a 1149 very small fraction (3-5\%), while only suffering a marginal 1150 increase in average cost per service component of $1-2 \%$ over 1151 the baseline algorithm, which, although possesses the least 1152 cost, suffers from a large number (up to 45\%) of deadline 1153 violations. Additionally, for time-varying ASAP workflows, 1154 the online clustering approach with a window size of 4 is able 1155 to restrict average deadline violations (per day) to $5-8 \%$ in comparison to that of (up to) $60 \%$ when the identified clusters 1156 were not updated over time. In the future, we will implement a 1157 $\mathrm{BRAHMA}^{+}$prototype running on real-world cloud platforms 1158 and evaluate its runtime behavior while scaling an elastic A/V ${ }_{1159}$ collaborative cloud-based service.

1160

\section{REFERENCES}

1161

[1] Amazon EC2 Images. Accessed: May 16, 2017. [Online]. Available: 1162 http://aws.amazon.com/ec2/instance-types/

[2] Amazon EC2 MIPS. Accessed: May 16, 2017. [Online]. Available: 1164 http://www.cmips.net/category/cup-results/

[3] Benchmarking the New Amazon C4 Instances. Accessed: May 16, 2017. 1166 [Online]. Available: http://www.cmips.net/tag/intel-xeon-e5-2666-v3-2- 1167 90ghz/

1168

[4] The EMD Project. Accessed: May 16, 2017. [Online]. Available: 1169 https://www.imec-int.com/en/what-we-offer/research-portfolio/emd 1170

[5] Tupperware: Containerized Deployment at Facebook. Accessed: 1171 May 16, 2017. [Online]. Available: http://bit.ly/2sD46ng 1172

[6] S. Abrishami and M. Naghibzadeh, "Deadline-constrained workflow 1173 scheduling in software as a service cloud," Scientia Iranica, vol. 19, 1174 no. 3, pp. 58-169, 2012.

1175

[7] A. F. Antonescu and T. Braun, "SLA-driven simulation of multi- 1176 tenant scalable cloud-distributed enterprise information system," in Proc. 1177 ARMS-CC@PODC, 2014,pp.91-102.

[8] A. Atrey, H. Moens, G. V. Seghbroeck, B. Volckaert, and F. D. Turck, 1179 "BRAHMA: An intelligent framework for automated scaling of stream- 1180 ing and deadline-critical workflows," in Proc. CNSM, Montreal, QC, 1181 Canada, 2016, pp. 216-222.

1182

[9] A. Atrey, H. Moens, G. V. Seghbroeck, B. Volckaert, and F. D. Turck, 1183 "Design and evaluation of automatic workflow scaling algorithms 1184 for multi-tenant SaaS," in Proc. CLOSER, Rome, Italy, 2016, 1185 pp. 221-229.

1186

[10] E. Boutin et al., "Apollo: Scalable and coordinated scheduling for 1187 cloud-scale computing," in Proc. OSDI, Broomfield, CO, USA, 2014, 1188 pp. 285-300.

1189

11] L. Breiman, "Random forests," Mach. Learn., vol. 45, no. 1, pp. 5-32, 1190 2001.

1191

[12] R. N. Calheiros, R. Ranjan, A. Beloglazov, C. A. F. D. Rose, and 1192 R. Buyya, "CloudSim: A toolkit for modeling and simulation of 1193 cloud computing environments and evaluation of resource provisioning 1194 algorithms," Softw. Pract. Exp., vol. 41, no. 1, pp. 23-50, 2011.

[13] J. Espadasa et al., "A tenant-based resource allocation model for 1196 scaling software-as-a-service applications over cloud computing infras- 1197 tructures," Future Gener. Comput. Syst., vol. 29, no. 1, pp. 273-286, 1198 2013.

[14] G. Fan, H. Yu, and L. Chen, "A formal aspect-oriented method for 1200 modeling and analyzing adaptive resource scheduling in cloud comput- 1201 ing," IEEE Trans. Netw. Service Manag., vol. 13, no. 2, pp. 281-294, 1202 Jun. 2016.

1203

[15] J. Gama, "Functional trees," Mach. Learn., vol. 55, no. 3, pp. 219-250, 1204 2004.

[16] T. A. L. Genez, L. F. Bittencourt, and E. R. M. Madeira, "Workflow 1206 scheduling for SaaS / PaaS cloud providers considering two SLA levels," 1207 in Proc. NOMS, 2012, pp. 906-912.

[17] M. H. Ghahramani, M. Zhou, and C. T. Hon, "Toward cloud com- 1209 puting QoS architecture: Analysis of cloud systems and cloud ser- 1210 vices," IEEE/CAA J. Automatica Sinica, vol. 4, no. 1, pp. 6-18, 1211 Jan. 2017.

1212

[18] M. Hall et al., "The WEKA data mining software: An update," SIGKDD 1213 Explor. Newslett., vol. 11, no. 1, pp. 10-18, 2009.

1214

[19] J. A. Hartigan and M. A. Wong, "Algorithm AS 136: A K-means 1215 clustering algorithm," Appl. Stat., vol. 28, no. 1, pp. 100-108, 1979. 1216

[20] R. Hyndman and G. Athanasopoulos, Forecasting: Principles and 1217 Practice. Melbourne, VIC, Australia: OTexts, 2013. [Online]. Available: 1218 https://www.otexts.org/fpp/

1219

[21] M. Isard, "Autopilot: Automatic data center management," SIGOPS 1220 Oper. Syst. Rev., vol. 41, no. 2, pp. 60-67, 2007.

[22] B. Jennings and R. Stadler, "Resource management in clouds: Survey 1222 and research challenges," J. Netw. Syst. Manag., vol. 23, no. 3, 1223 pp. 567-619, 2015.

[23] H.-J. Jiang, K.-C. Huang, H.-Y. Chang, D.-S. Gu, and P.-J. Shih, 1225 "Scheduling concurrent workflows in HPC cloud through exploiting 1226 schedule gaps," in Proc. ICA3PP, Melbourne, VIC, Australia, 2011, 1227 pp. 282-293. 
229 [24] J. Li, T. Ma, M. Tang, W. Shen, and Y. Jin, "Improved FIFO scheduling algorithm based on fuzzy clustering in cloud computing," Information, vol. 8 , no. 1 , p. 25,2017

25] H. Lu, J. Cao, S. Lv, X. Wang, and J. Liu, "A comparative study of DAG clustering," in Proc. i-Soc., London, U.K., 2015, pp. 84-89.

26] H. Luo, C. Yan, and Z. Hu, "An enhanced workflow scheduling strategy for deadline guarantee on hybrid grid/cloud infrastructure," J. Appl. Sci. Eng., vol. 18, no. 1, pp. 67-78, 2015.

[27] M. Mao and M. Humphrey, "A performance study on the VM startup time in the cloud," in Proc. IEEE CLOUD, Honolulu, HI, USA, 2012, pp. 423-430.

28] E. E. Mon, M. M. Thein, and M. T. Aung, "Clustering based on task dependency for data-intensive workflow scheduling optimization," in Proc. MTAGS, Salt Lake City, UT, USA, 2016, pp. 20-25.

[29] H. Morshedlou and M. R. Meybodi, "Decreasing impact of SLA violations: A proactive resource allocation approach for cloud computing environments," IEEE Trans. Cloud Comput., vol. 2, no. 2, pp. 156-167, Apr./Jun. 2014.

30] G. Peng, H. Wang, J. Dong, and H. Zhang, "Knowledge-based resource allocation for collaborative simulation development in a multi-tenan cloud computing environment," IEEE Trans. Services Comput., vol. 11, no. 2, pp. 306-317, Mar./Apr. 2018.

31] D. Poola, S. K. Garg, R. Buyya, Y. Yang, and K. Ramamohanarao, "Robust scheduling of scientific workflows with deadline and budget constraints in clouds," in Proc. IEEE-AINA, Victoria, BC, Canada, 2014, pp. 858-865.

32] D. Poola, K. Ramamohanarao, and R. Buyya, "Enhancing reliability of workflow execution using task replication and spot instances," Trans. Auton. Adapt. Syst., vol. 10, no. 4, pp. 1-21, 2016.

33] J. R. Quinlan, C4.5: Programs for Machine Learning. Burlington, MA, USA: Morgan Kaufmann, 1993.

[34] M. A. Rodriguez and R. Buyya, "Deadline based resource provisioning and scheduling algorithm for scientific workflows on clouds," IEEE Trans. Cloud Comput., vol. 2, no. 4, pp. 222-235, Apr./Jun. 2014.

35] M. A. Rodriguez and R. Buyya, "Scheduling dynamic workloads in multi-tenant scientific workflow as a service platforms," Future Gener Comput. Syst., vol. 79, pp. 739-750, Feb. 2017.

36] P. J. Rousseeuw, "Silhouettes: A graphical aid to the interpretation and validation of cluster analysis," J. Comput. Appl. Math., vol. 20, pp. 53-65, Nov. 1987.

[37] D. Serrano et al., "SLA guarantees for cloud services," Future Gener. Comput. Syst., vol. 54, pp. 233-246, Jan. 2016.

[38] J. Shi, J. Luo, F. Dong, J. Zhang, and J. Zhang, "Elastic resource provisioning for scientific workflow scheduling in cloud under budget and deadline constraints," Cluster Comput., vol. 19, no. 1, pp. 167-182, 2016.

39] S. Singh, I. Chana, and R. Buyya, "STAR: SLA-aware autonomic management of cloud resources," IEEE Trans. Cloud Comput., to be published.

[40] S. Toyoshima, S. Yamaguchi, and M. Oguchi, "Storage access optimization with virtual machine migration and basic performance analysis of Amazon EC2," in Proc. WAINA, Perth, WA, Australia, 2010, pp. 905-910.

1] D. Tuncer, M. Charalambides, S. Clayman, and G. Pavlou, "Adaptive resource management and control in software defined networks," IEEE Trans. Netw. Service Manag., vol. 12, no. 1, pp. 18-33, Mar. 2015.

[42] A. Verma et al., "Large-scale cluster management at Google with Borg," in Proc. EuroSys, Bordeaux, France, 2015, p. 18.

3] F. Wu, Q. Wu, and Y. Tan, "Workflow scheduling in cloud: A survey," J. Supercomput., vol. 71, no. 9, pp. 3373-3418, 2015.

4] L. Wu, S. K. Garg, S. Versteeg, and R. Buyya, "SLA-based resource provisioning for hosted software-as-a-service applications in cloud computing environments," IEEE Trans. Services Comput., vol. 7, no. 3 pp. 465-485, Jul./Sep. 2014.

5] L. Wu, S. K. Garg, and R. Buyya, "SLA-based resource allocation for software as a service provider (SaaS) in cloud computing environments," in Proc. CCGrid, Newport Beach, CA, USA, 2011, pp. 195-204.

6] L. Wu, S. K. Garg, and R. Buyya, "SLA-based admission control for a software-as-a-service provider in cloud computing environments," $J$. Comput. Syst. Sci., vol. 78, no. 5, pp. 1280-1299, 2012.

[47] R. Zhang, K. Wu, and J. Wang, "Online resource scheduling under concave pricing for cloud computing," in Proc. IWQoS, Hong Kong, 2014, pp. 51-60.

48 ] Z. Zhang et al., "Fuxi: A fault-tolerant resource management and job scheduling system at Internet scale," Proc. VLDB Endowment, vol. 7, no. 13 , pp. 1393-1404, 2014.

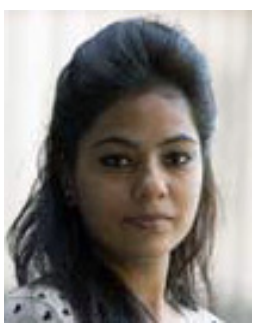

Ankita Atrey received the master's degree in 1306 computer science from the Vellore Institute of 1307 Technology, Vellore, India. She is currently pur- 1308 suing the Ph.D. degree with the Department of 1309 Information Technology (INTEC), Ghent University, 1310 Belgium, and imec. She has internship experience 1311 from CNRS, France, and the Indian Institute of 1312 Technology Kanpur, India. Her research interests 1313 include cloud computing, resource scheduling and 1314 provisioning, data-placement, service management, 1315 and service oriented architectures. She is working on 1316 research problems encircling intelligent resource provisioning in multi-tenant 1317 multi-component applications with INTEC. She has published her research in 1318 cloud and service management conferences like CNSM and CLOSER, while 1319 also serving as a reviewer for CLOSER, Journal of Network and Systems 1320 Management and the IEEE TRANSACTION ON NETWORK AND SERVICE 1321 MANAGEMENT.

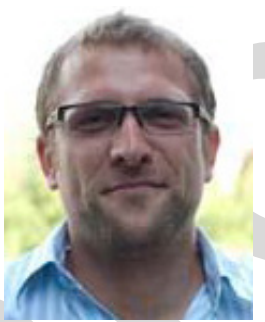

Gregory Van Seghbroeck received the gradua- 1323 tion degree from Ghent University in 2005 and the 1324 Ph.D. degree in computer science engineering in 1325 2011. After a brief time as an IT Consultant, he 1326 joined the Department of Information Technology 1327 (INTEC), Ghent University (currently IDLab). In 1328 2007, he received the Ph.D. grant from IWT, 1329 Institute for the Support of Innovation Through 1330 Science and Technology, to work on theoretical 1331 aspects of advanced validation mechanism for dis- 1332 tributed interaction protocols and service choreogra- 1333 phies. His main research interests focus on big data engineering and complex 1334 scalable cloud platforms.

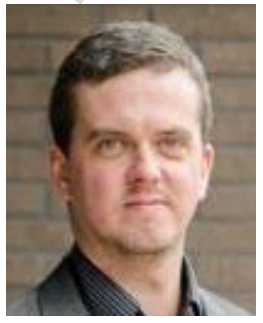

Bruno Volckaert received the Master of Computer 1336 Science degree and the Ph.D. degree in data inten- 1337 sive scheduling and service management for grid 1338 computing from Ghent University, in 2001 and 1339 2006, respectively. He is a Professor of advanced 1340 programming and software engineering with the 1341 Department of Information Technology (INTEC), 1342 Ghent University and a Senior Researcher with imec. 1343 His current research deals with reliable and high 1344 performance distributed software systems for City- 1345 of-Things (IoT for Smart Cities), distributed decision 1346 support systems for UAVs, intelligent railway transportation applications and 1347 autonomous optimization of cloud-based applications. He has worked on over 1348 35 national and international research projects and has authored or co-authored 1349 over 80 papers published in international journals and conference proceedings. 1350

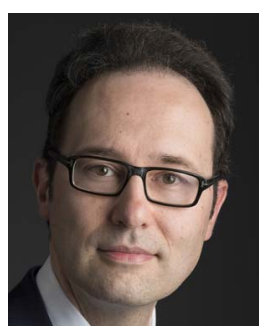

Filip De Turck leads the Network and Service 1351 Management Research Group, Department of 1352 Information Technology, Ghent University, 1353 Belgium, and imec. He has (co-)authored over 1354 450 peer reviewed papers. His research interests 1355 include telecommunication network and service 1356 management, and design of efficient virtualized 1357 network and cloud systems. He is involved in 1358 several research projects with industry and 1359 academia in the above areas. He serves as the 1360 Chair of the IEEE Technical Committee on 1361 Network Operations and Management, and is on the TPC of many 1362 network and service management conferences and workshops. He serves 1363 as a Steering Committee Member of the IEEE Conference on Network 1364 Softwarization. 